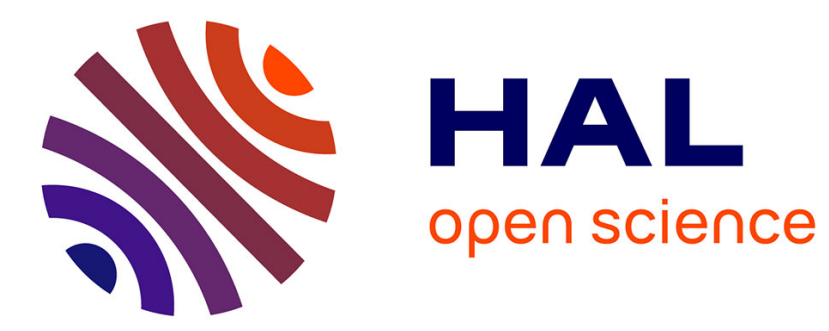

\title{
Flower-Visiting Records of the Native Bees of New Caledonia
}

Barry Donovan, Jérôme Munzinger, Alain Pauly, Gordon Mcpherson

\section{To cite this version:}

Barry Donovan, Jérôme Munzinger, Alain Pauly, Gordon Mcpherson. Flower-Visiting Records of the Native Bees of New Caledonia. Annals, 2013, 99 (1), pp.19-43. 10.3417/2010076 . hal-02945907

\section{HAL Id: hal-02945907 https://hal.inrae.fr/hal-02945907}

Submitted on 22 Sep 2020

HAL is a multi-disciplinary open access archive for the deposit and dissemination of scientific research documents, whether they are published or not. The documents may come from teaching and research institutions in France or abroad, or from public or private research centers.
L'archive ouverte pluridisciplinaire HAL, est destinée au dépôt et à la diffusion de documents scientifiques de niveau recherche, publiés ou non, émanant des établissements d'enseignement et de recherche français ou étrangers, des laboratoires publics ou privés. 


\title{
FLOWER-VISITING RECORDS OF Barry J. Donovan, ${ }^{2}$ Jérôme Munzinger, ${ }^{3}$ Alain THE NATIVE BEES OF NEW $\quad$ Pauly, ${ }^{4}$ and Gordon McPherson ${ }^{5}$ CALEDONIA $^{1}$
}

\begin{abstract}
The flower-visiting records for the 43 species of bees considered to be native to New Caledonia show that females of 21 species visited 116 native species of plants in 69 genera and 41 families, and the bees were documented to carry pollen from 64 species and possibly four more. The plant families with the greatest number of species documented for visits by female bees were, in descending order: Myrtaceae (21), Dilleniaceae (10), Cunoniaceae (nine), Araliaceae (seven), Fabaceae (seven, encompassing the Caesalpinioideae, Mimosoideae, and Papilionoideae), Goodeniaceae (five), Proteaceae (five), Apocynaceae (four), Sapindaceae (four), and remaining families with one to three species. Females of six and possibly one more species carried pollen from each of Dilleniaceae and Myrtaceae, six carried pollen from Araliaceae, five from Goodeniaceae, four and possibly one more from Cunoniaceae, four from each of Fabaceae and Sapindaceae, and none to three from the remaining 34 families observed. For introduced plants, female bees of 12 species visited 54 species in 43 genera among 19 plant families and were documented to carry pollen from 31 and possibly one more species. For introduced plants, families with the highest number of species visited by female bees, in descending order, were: Asteraceae (12); Fabaceae (eight); Verbenaceae (seven); and Euphorbiaceae, Myrtaceae, and Solanaceae each with three. The remaining 13 families had either one or two species visited by bees. Females of seven species of bees carried pollen from Fabaceae, six from Asteraceae, three each from Myrtaceae and Solanaceae, and none to " $2+1$ ?" (two or possibly three) from remaining families. Only half a dozen species of native bees can be considered to be common, in that they can be expected to be observed reasonably regularly on a range of flowers. The ubiquitous introduced honey bee Apis mellifera L. and its constant foraging for nectar and pollen on a very wide range of flowers may outcompete many species of native bees, potentially reducing their numbers, and consequently obscuring their relationships with the flora.

Key words: Apis, Araliaceae, competitive foraging pressure, Cunoniaceae, Dilleniaceae, Fabaceae, flower-visiting records, Goodeniaceae, introduced honey bee, Myrtaceae, native bees, New Caledonia, Proteaceae.
\end{abstract}

For a land mass of $19,103 \mathrm{~km}^{2}$, New Caledonia is considered unique based on the high number of flowering plant species (3051) and its high endemicity $(77.7 \%$; Morat et al., 2012), which markedly contrasts with the low number of bee species (28 species, Donovan, 1983; 21 species, Pauly \& Munzinger, 2003). A major question for evolutionary biologists is how so many distinctive species of flowering plants could have evolved with so few apparent pollinating bees. This includes a relative paucity of other pollinating insects, birds, and reptiles (Kato \& Kawakita, 2004). In contrast, the number of known species of bees has about doubled in recent years. Taxonomic revisions of the major bee groups are currently underway, but will be time consuming, and yet there is much interest in the relationships between the various species of bees and flowering plants. The purpose of this study is to make available now all bee flower-visiting data. Observations for Apis mellifera L. are explicitly excluded because this honey bee was purposely introduced in 1848 (Lamaignere, 2001) and is not a naturally occurring constituent of the flower-visiting Apoidea. The term "native" for the remaining species of bees is used here to indicate those species that were likely present before the arrival of any humans about several thousand years ago (Irwin, 2010), and also those that might not have been knowingly introduced by humans. However, in the absence of intensive surveys of the bees of lands of the Southwest Pacific,

\footnotetext{
${ }^{1}$ We thank the Missouri Botanical Garden and the John D. and Catherine T. MacArthur Foundation for funding bee and plant collecting on New Caledonia by Gordon McPherson, Barry Donovan, Jason Bradford, and Helen Fortune, and we thank Jason Bradford and Helen Fortune for some plant identifications. Some bees were collected in the Kouakoué-Ni reserve during a botanical assessment supported by a National Geographic Society grant $N^{\circ} 7579-04$ to Jérôme Munzinger. The preparation of this manuscript was suggested by Dr. Peter Raven. Finally, we thank North and South Environmental Services in New Caledonia for collecting permits and logistic support.

2 Donovan Scientific Insect Research, Canterbury Agriculture and Science Centre, Lincoln, Private Bag 4704, Christchurch, New Zealand. Barry.Donovan@Plantandfood.co.nz.

3 UMR AMAP, Laboratoire de Botanique et d'Écologie Végétale Appliquées, Herbarium NOU, F-98848 NouvelleCalédonie; IRD, UMR AMAP, Montpellier, F-34000 France. Jerome.Munzinger@ird.fr.

${ }^{4}$ Institut Royal des Sciences Naturelles des Belgique, Département d'Entomologie, Rue Vautier 29, B-1000 Brussels, Belgium. Alain_Pauly@brutele.be.

5 Missouri Botanical Garden, P.O. Box 299, St. Louis, Missouri 63166-0299, U.S.A. Gordon.McPherson@mobot.org. doi: $10.3417 / 2010076$
} 


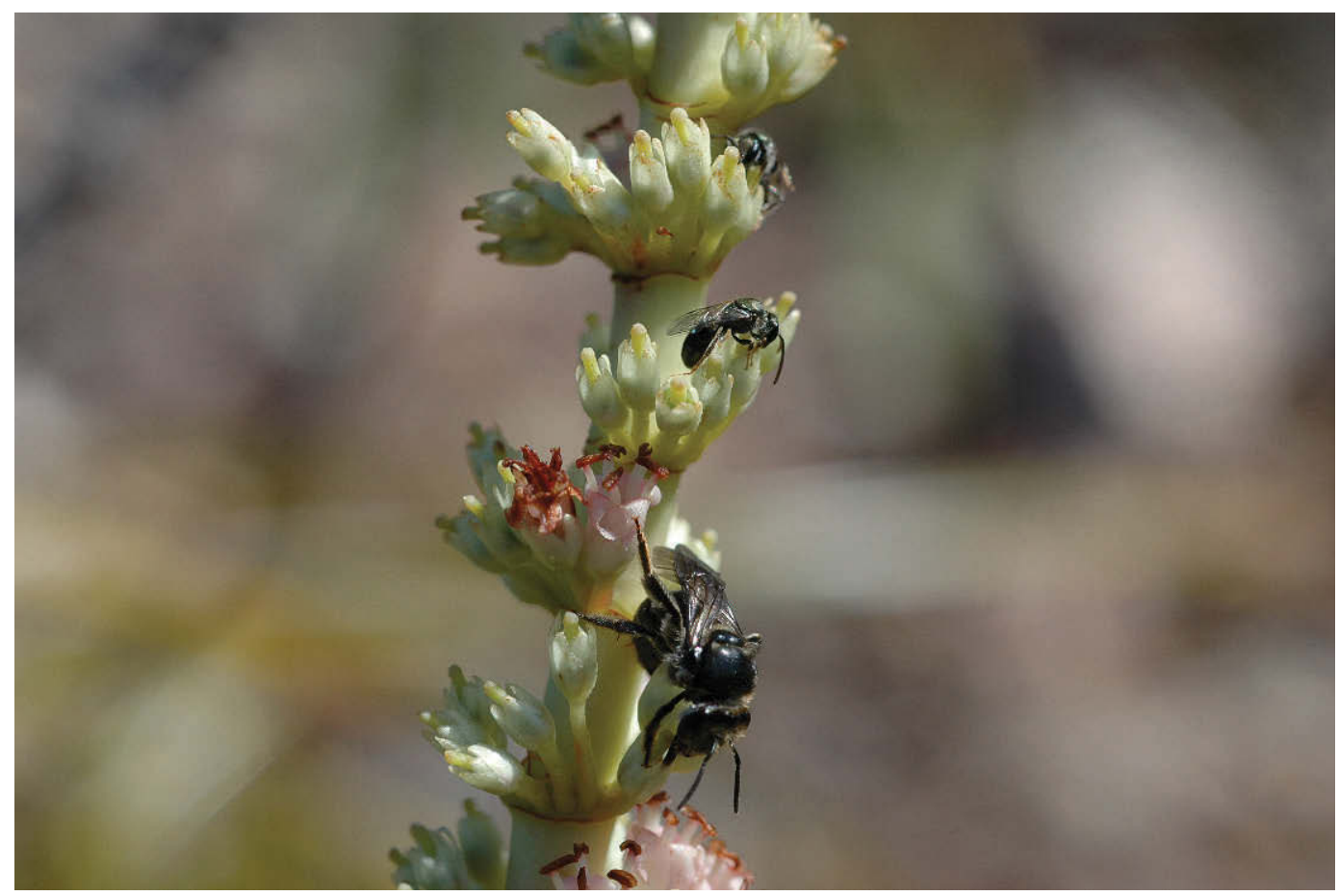

Figure 1. Two female Homalictus risbeci (Cockerell, 1929) (the two small bees) and a female Austronomia sicheli (Vachal, 1897) (the large bee) visiting the flowers of Dracophyllum verticillatum Labill (Ericaceae), Col d'Arama, New Caledonia, 29 July 2005. Photograph by J. Munzinger.

the true status of many species may be uncertain (Groom \& Schwarz, 2011).

\section{Methods}

Bees from New Caledonia from all known collections worldwide were borrowed, and flower data were recorded from labels attached to the pins. In addition, bees were collected with insect nets from flowers on Grande Terre during December 1979 and April to May 1981, and Grande Terre and Ile des Pins during October 1980 by B. J. Donovan and G. McPherson; on Grande Terre during January and May 1999, March to April 2001, November to December 2001, November to December 2002, and January 2004 by J. Munzinger; on Grande Terre during November to December 2000 by B. J. Donovan, H. Fortune, and J. Bradford; and Grande Terre and Lifou during July to August 2003 by B. J. Donovan. Since April 2004 to September 2008, bees have been collected at various times of year throughout New Caledonia by J. Munzinger (Fig. 1). The identity of flowers from which bees were collected was recorded on site, or if the flower could not be identified, specimens were taken for later study and identification; these vouchers were deposited in the herbaria of MO and NOU.
Bees were examined microscopically and where possible were identified to species, or if identification was not possible, numbered as separate taxa. The names of species of bees are as in Michener (2007), with the exception of the insect genus Lipotriches Gerstaecker, which is now referred to as Austronomia Michener. In Tables 1 and 3, the families, genera, and species of bees are ordered as in Michener (2007). As revisions of groups of bees are published (Donovan, in prep.; Pauly, in prep.), references in those works will be made to the species that are numbered in this study. For all species of bees, holotypes were examined wherever possible, and for species of bees named from areas beyond New Caledonia, voucher specimens were examined from New Caledonia. Data for voucher specimens are presented after the holotype data. Female bees were examined microscopically for evidence of pollen in scopae. When pollen is abundant the bee has obviously been collecting pollen for a while, but if a bee has just begun collecting pollen, very little may be evident. If scopae held very little pollen and it was no more abundant than on other parts of the bee, the bee was not considered to have been collecting pollen. However, if the pollen in scopae was at least partly clumped, the bee was considered to have been 
collecting pollen. Female hylaeine and euryglossine bees carry pollen internally, so if they were captured in association with flowers, whether they were collecting pollen could not be readily ascertained. Females of an undescribed cleptoparasitic species, which is referred to in Tables 1 and 3 as "Lasioglossum sp. indet. 9," do not carry pollen. Notations such as " $2 / 5$ p" (Table 1) indicate that there were two collections of a total of five bees, all of which were carrying pollen, while " $2 / 5$ (1/3 p)" refers to two collections of a total of five bees, of which one collection of three bees was carrying pollen. The notation " $0 / \mathrm{l}$ " indicates that one bee was observed on a flower. In this article, a notation such as " $4+$ 1?" means that females of four species of bees were carrying pollen, and possibly also the females of one species that carry pollen internally. A "collection" refers to an event during which bees were captured, and differs from another "collection" in at least one datum, such as the collector, the host plant, the site, or time of day. Because bees can fly rapidly, will sometimes visit a range of flowers, and may take just nectar and not also pollen from a flower, the collection from a particular flower of a bee that is carrying pollen does not necessarily mean that the pollen is from that flower. Microscopic examination of pollen on bees to identify the source of the pollen was beyond the scope of this study.

Female bees that collect much pollen from the flowers of a particular species of plant are often considered to be pollinating the flowers, but their true status as pollinators can be determined only by assessing the number of pollen grains deposited on stigmas. In contrast to female bees, male bees do not collect pollen to carry to nests, and they visit flowers comparatively fleetingly only to sup nectar and perhaps to feed on pollen grains. Compared to female bees, male bees are therefore far less likely to be effective pollinators.

Native plant names follow Jaffré et al. (2004), or the updated version of this checklist (Morat et al., 2012). Information presented here includes sources such as Donovan (1983), Pauly and Munzinger (2003), Nielsen et al. (2005), and Kato and Kawakita (2004).

\section{Results}

Forty-three species of native bees were identified from New Caledonia, and of these the females of 21 species visited the flowers of 116 native species of plants in 69 genera and 41 families (Tables 1 and 2). Twenty-one of these plant species belonged to the Myrtaceae, 10 to Dilleniaceae, nine to Cunoniaceae, seven each to Araliaceae and Fabaceae s.l. (including the Caesalpinioideae, Mimosoideae, and Papilionoideae), five each to Goodeniaceae and Proteaceae, and four each to Apocynaceae and Sapindaceae, while for the remaining families the number of species ranged from one to three. Species in Dilleniaceae and Myrtaceae were each visited by females of nine species of bees; Araliaceae by eight; Cunoniaceae and Ericaceae each by seven species; Goodeniaceae by six species; Arecaceae, Fabaceae, Proteaceae, and Violaceae each by five species; and the remaining families were visited by one to four species. Females of " $6+1$ ?" species of bees carried pollen from Dilleniaceae and Myrtaceae, six from Araliaceae, five from Goodeniaceae, " $4+1$ ?" from Cunoniaceae, four each from Fabaceae and Sapindaceae, and none to three from the remaining 34 families. Bees carried pollen from " $64+4$ ?" plant species, of which 11 belonged to Myrtaceae; six to Fabaceae; " $5+2$ ?" to Dilleniaceae; " $4+1$ ?" to Cunoniaceae; four to each of Araliaceae, Goodeniaceae, Proteaceae, and Sapindaceae; and none to two for the remaining families.

For introduced plants, female bees of 12 species visited 54 species in 43 genera and 19 families (Tables 1 and 2). Twelve of the plant species belonged to Asteraceae; eight to Fabaceae; seven to Verbenaceae; three each to Euphorbiaceae, Myrtaceae, and Solanaceae; and from one to two for the remaining families. Species in Fabaceae were visited by females of eight species of bees, Asteraceae and Verbenaceae by seven, Sapindaceae by four, and the remaining families by one to three. The females of seven species of bees visiting Fabaceae carried pollen, six carried pollen from Asteraceae, three carried pollen from each of Myrtaceae and Solanaceae, while none to " $2+$ 1?" carried pollen from the remaining families. Pollen was carried by female bees from six species of plants in both Asteraceae and Fabaceae, three in Solanaceae, and none to two in the remaining families.

One species each of Asteraceae and Convolvulaceae are of uncertain identity (Table 2) and were visited by females of three species of bees.

For species of bees with females that visited the flowers of native plants, of the six species with more than 10 records, Austronomia sicheli Vachal visited most species (53 spp.) and carried pollen from the highest number (29 spp.) (Table 3). However, for introduced plants only three species of bees visited 10 or more species, of which Homalictus aponi Cheesman \& Perkins visited the most species (24 spp.) and carried pollen from the most species (14 spp.).

\section{Discussion}

Although the 43 native species of bees now known from New Caledonia are nearing double the mean of 
Table 1. Alphabetic list of families, subfamilies, genera, and species of bees, bee voucher and bee collection data, and the vascular plants associated with each bee species.

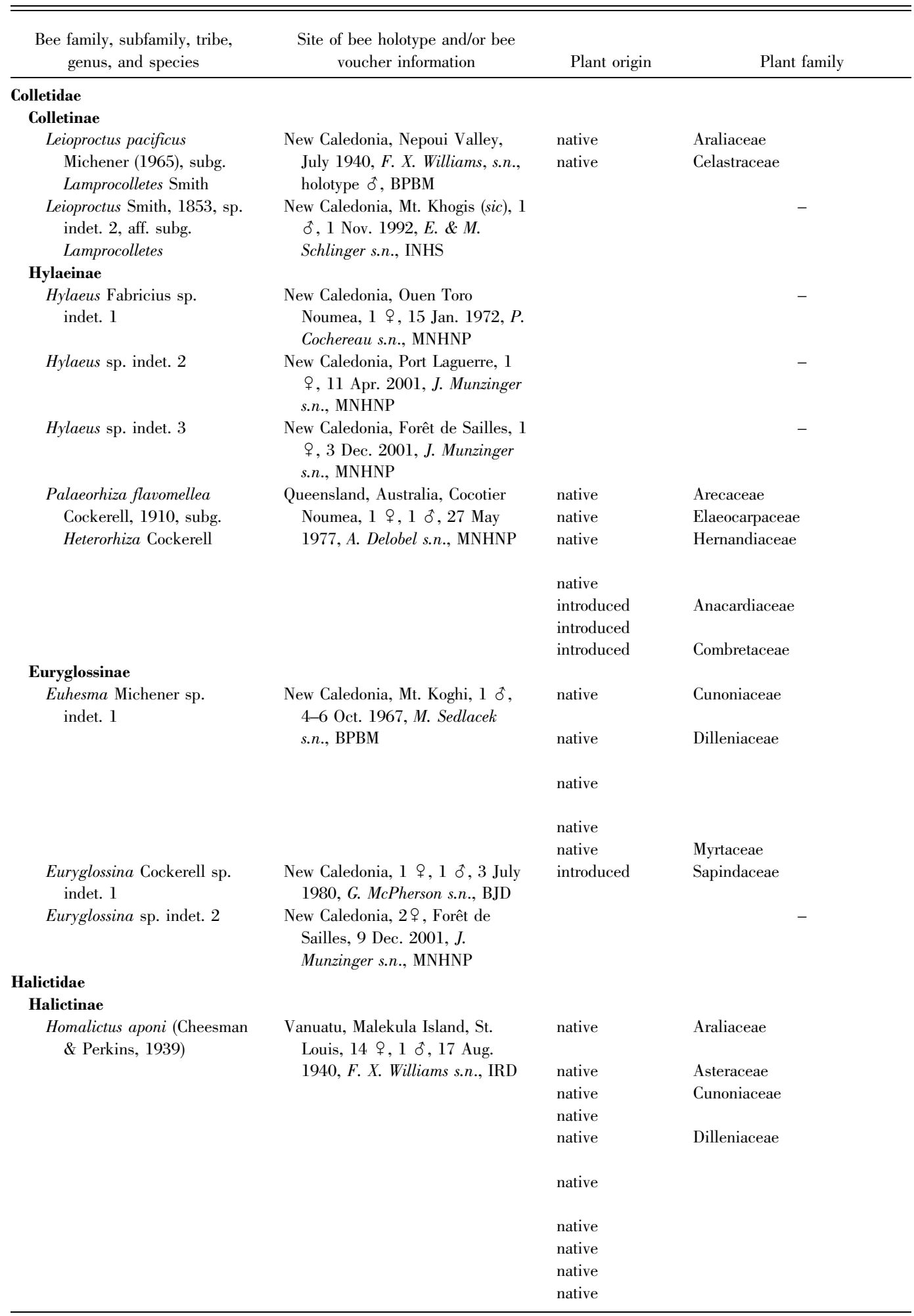


Table 1. Extended.

\begin{tabular}{|c|c|c|c|}
\hline \multirow[b]{2}{*}{ Plant species } & \multirow[b]{2}{*}{ Plant voucher information } & \multicolumn{2}{|c|}{ Bee collection data } \\
\hline & & q & o \\
\hline
\end{tabular}

Myodocarpus fraxinifolius Brongn. \& Gris Peripterygia marginata (Baill.) Loes.

Peripterygia marginata (Baill.) Loes.

Cocos nucifera L.

Hibbertia pulchella (Brongn. \& Gris) 
Table 1. Continued.

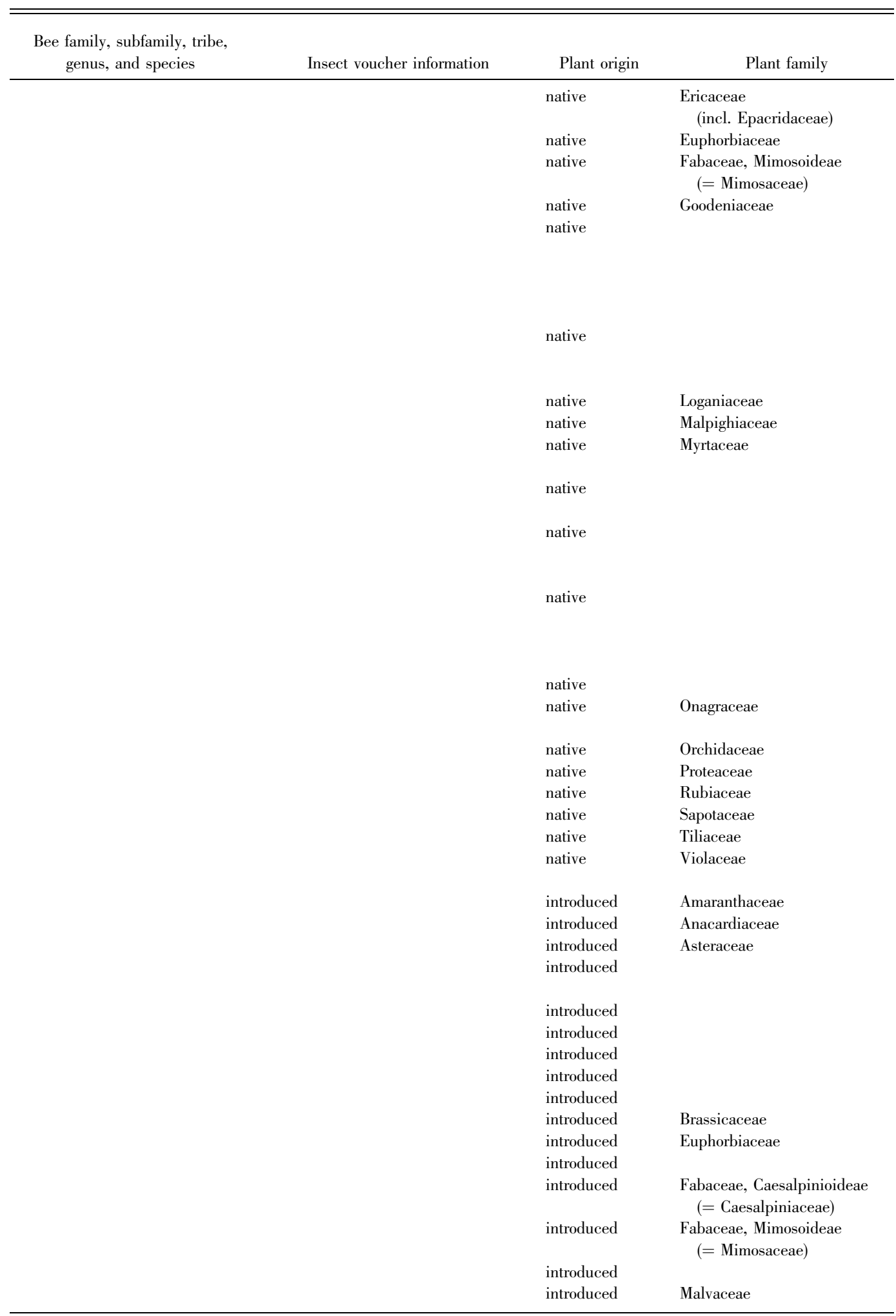


Table 1. Continued, Extended.

\begin{tabular}{|c|c|c|c|}
\hline \multirow[b]{2}{*}{ Plant species } & \multirow[b]{2}{*}{ Plant voucher information } & \multicolumn{2}{|c|}{ Bee collection data } \\
\hline & & q & $\hat{0}$ \\
\hline $\begin{array}{l}\text { Styphelia Sm. sp. indet., cf. S. cymbulae } \\
\text { (Labill.) Spreng. }\end{array}$ & & $\uparrow 1 / 1$ & - \\
\hline sp. indet. & & ㅇ $1 / 1$ & o $1 / 4$ \\
\hline Acacia spirorbis Labill. & & $4 / 13(4 / 8 \mathrm{p})$ & ô $1 / 1$ \\
\hline Scaevola beckii Zahlbr. & & q $2 / 2 \mathrm{p}$ & - \\
\hline Scaevola montana Labill. & 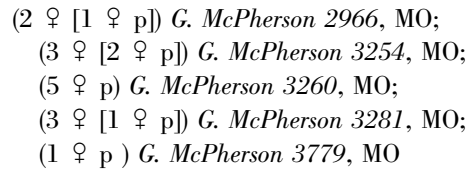 & ㅇ 9/18 (7/11 p) & ô $1 / 1$ \\
\hline Scaevola L. sp. indet. & $\begin{array}{l}J . \text { Munzinger } 864, \text { NOU; J. Munzinger } \\
\text { 1382, NOU; }(2 \text { \&) J. Munzinger } 2958, \\
\text { NOU }\end{array}$ & ㅇ 4/4 (1/1 p) & - \\
\hline Geniostoma densiflora Baill. & G. McPherson 3282, MO & ㅇ $1 / 1$ & - \\
\hline Acridocarpus austrocaledonicus Baill. & J. C. Bradford 1053, МO & q $1 / 1 \mathrm{p}$ & - \\
\hline $\begin{array}{l}\text { Melaleuca quinquenervia (Cav.) S. T. } \\
\text { Blake }\end{array}$ & & $q 2 / 5 \mathrm{p}$ & - \\
\hline $\begin{array}{l}\text { Metrosideros operculata Labill. var. } \\
\text { operculata }\end{array}$ & G. McPherson 3786, MO & q $1 / 1$ & - \\
\hline $\begin{array}{l}\text { Sannantha leratii (Schltr.) Peter G. Wilson } \\
\text { [三 Babingtonia leratii (Schltr.) A. R. } \\
\text { Bean] }\end{array}$ & & q 4/11 (1/2 p) & - \\
\hline $\begin{array}{l}\text { Sannantha virgata (J. R. Forst. \& G. } \\
\text { Forst.) Peter G. Wilson [三 Babingtonia } \\
\text { virgata (J. R. Forst. \& G. Forst.) F. } \\
\text { Muell.] }\end{array}$ & J. Munzinger 2652, NOU & q $1 / 1$ & - \\
\hline Xanthostemon F. Muell. sp. indet. & & - & o $1 / 1$ \\
\hline $\begin{array}{l}\text { Ludwigia octovalvis (Jacq.) P. H. Raven } \\
\text { subsp. octovalvis }\end{array}$ & (2 + p) G. McPherson 3785, MO & ㅇ $2 / 3(1 / 2 \mathrm{p})$ & - \\
\hline Eriaxis rigida Rchb. f. & & q $1 / 1$ & - \\
\hline Stenocarpus phyllodineus S. Moore & G. McPherson 3317, MO & o $1 / 1 \mathrm{p}$ & - \\
\hline Normandia neocaledonica Hook. f. & & ㅇ $1 / 1$ & - \\
\hline Leptostylis petiolata Vink & & - & ô $1 / 1$ \\
\hline Corchorus L. sp. indet. & & ㅇ $1 / 1$ & - \\
\hline $\begin{array}{l}\text { Agatea longipedicellata (Baker f.) } \\
\text { Guillaumin \& Thorne }\end{array}$ & J. Munzinger $320, \mathrm{NOU}$ & ㅇ $1 / 1(1 / 1 \mathrm{p})$ & - \\
\hline Achyranthes aspera L. & G. McPherson 3246, MO & $1 / 1$ & - \\
\hline Schinus terebinthifolia & & - & ô $1 / 1$ \\
\hline Bidens pilosa L. & & ㅇ $1 / 1$ & ô $1 / 1$ \\
\hline $\begin{array}{l}\text { Conyza Less. sp. indet., cf. C. bonariensis } \\
\text { (L.) Cronquist }\end{array}$ & & ๆ $1 / 1$ & - \\
\hline Calendula L. sp. indet. & & ๆ $1 / 1 \mathrm{p}$ & - \\
\hline Emilia sonchifolia (L.) DC. & 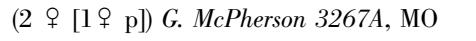 & ㅇ $3 / 4(1 / 1 \mathrm{p})$ & - \\
\hline Tridax procumbens $\mathrm{L}$. & & ㅇ $3 / 13(2 / 12 \mathrm{p})$ & - \\
\hline Tridax L. sp. indet. & & ㅇ $1 / 11(1 / 6 \mathrm{p})$ & ô $1 / 2$ \\
\hline Youngia japonica (L.) DC. & & ㅇ $1 / 1$ & - \\
\hline Lepidium virginicum $\mathrm{L}$. & & ㅇ $1 / 1$ & - \\
\hline Euphorbia hypericifolia L. & & 우 $4 / 4(3 / 4 \mathrm{p})$ & ○ $5 / 5$ \\
\hline Euphorbia lophogona Lam. & & ㅇ $1 / 1 \mathrm{p}$ & - \\
\hline Crotalaria L. sp. indet. & J. Munzinger 445, NOU & $q 1 / 1$ & - \\
\hline Leucaena leucocephala (Lam.) de Wit & 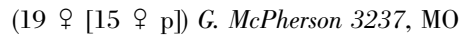 & $4 / 30(4 / 24 p)$ & - \\
\hline Mimosa diplotricha C. Wright & (5 \& p) G. McPherson 3776, MO & ㅇ $2 / 6 \mathrm{p}$ & - \\
\hline Sida acuta Burm. f. & & ㅇ $1 / 5(1 / 4 \mathrm{p})$ & ô $1 / 3$ \\
\hline
\end{tabular}


Table 1. Continued.

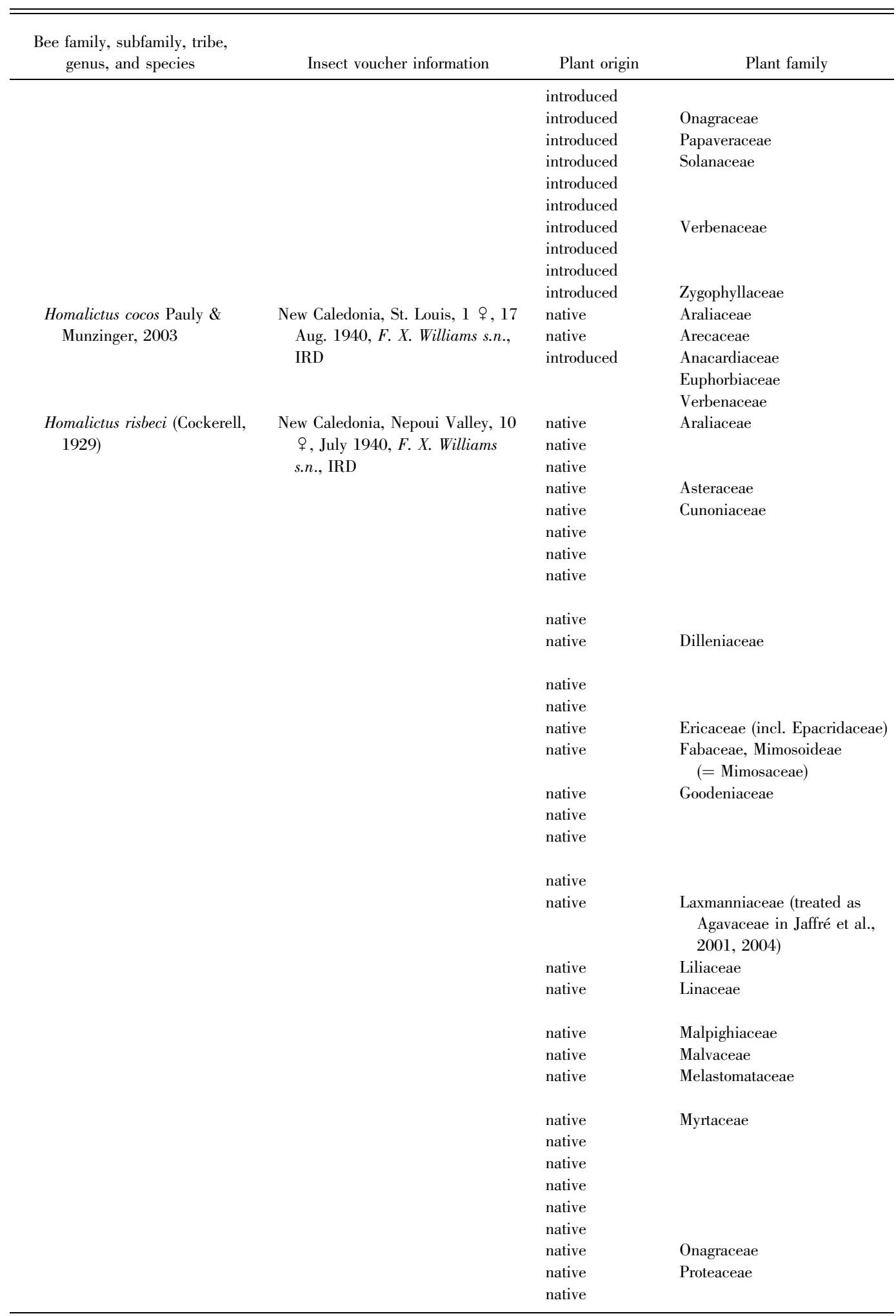


Table 1. Continued, Extended.

\begin{tabular}{|c|c|c|c|}
\hline \multirow[b]{2}{*}{ Plant species } & \multirow[b]{2}{*}{ Plant voucher information } & \multicolumn{2}{|c|}{ Bee collection data } \\
\hline & & o & $\hat{0}$ \\
\hline Sida rhombifolia $\mathrm{L}$. & & q 7/7 & - \\
\hline Bougainvillea Comm. ex Juss. sp. indet. & & - & ô $1 / 1$ \\
\hline Argemone mexicana $\mathrm{L}$. & G. McPherson 3255, MO & ㅇ $2 / 16(2 / 9 \mathrm{p})$ & - \\
\hline Solanum lycopersicum L. & J. Munzinger 609, NOU & ㅇ $2 / 2(1 / 1 \mathrm{p})$ & - \\
\hline Solanum torvum Sw. & & 우 $1 / 9(1 / 6 \mathrm{p})$ & - \\
\hline Solanum L. sp. indet. & & ㅇ $1 / 1$ & - \\
\hline Duranta repens $\mathrm{L}$. & & @ $1 / 1$ & ô $1 / 1$ \\
\hline Stachytarpheta indica (L.) Vahl & & ᄋ $1 / 1$ & ô $1 / 8$ \\
\hline Verbena L. sp. indet. & & ㅇ $1 / 11(1 / 7 \mathrm{p})$ & ô $1 / 1$ \\
\hline Tribulus cistoides $\mathrm{L}$. & & q 9/15 (6/12 p) & ô $1 / 1$ \\
\hline Meryta J. R. Forst. \& G. Forst. sp. indet. & & q $1 / 1$ & - \\
\hline Cocos nucifera & & 우 $2 / 2$ & - \\
\hline Schinus terebinthifolia & & 우 $2 / 3(2 / 2 \mathrm{p})$ & ô $2 / 12$ \\
\hline Poinsettia Graham sp. indet. & & ㅇ $3 / 3$ & ô $1 / 1$ \\
\hline Verbena sp. indet. & & ㅇ $1 / 1$ & - \\
\hline Polyscias sessiliflora Marais subsp. indet. & & - & ô $1 / 1$ \\
\hline Polyscias sp. indet. & & 우 2/6 (2/5 p) & ○े $2 / 9$ \\
\hline genus and sp. indet. & & - & ô $1 / 1$ \\
\hline Blumea lacera & & ㅇ $1 / 1$ & - \\
\hline Geissois sp. indet. & & - & ô $1 / 1$ \\
\hline Pancheria alaternoides Brongn. \& Gris & J. Munzinger 2818, NOU & 우 $1 / 1$ & - \\
\hline Pancheria billardierei & & ㅇ $1 / 1$ & - \\
\hline $\begin{array}{l}\text { Pancheria phylliraeoides Brongn. \& Gris } \\
\text { ex Guillaumin }\end{array}$ & G. McPherson 3339, MO & $\uparrow 1 / 6 \mathrm{p}$ & - \\
\hline Pancheria Brongn. \& Gris sp. indet. & & - & ô $1 / 1$ \\
\hline Hibbertia lucens & $\begin{array}{l}\text { (2 \&) G. McPherson } 3272, \mathrm{MO} ;(1 \text { } ~ J . \\
\text { Munzinger } 959, \mathrm{NOU}\end{array}$ & $\uparrow 2 / 3 \mathrm{p}$ & - \\
\hline Hibbertia pancheri (Brongn. \& Gris) Briq. & & $q 1 / 1$ & - \\
\hline Hibbertia sp. indet. & & q $1 / 1$ & - \\
\hline Dracophyllum verticillatum Labill. & J. Munzinger 2957, NOU & ㅇ $0 / 2$ & o $4 / 4$ \\
\hline Acacia spirorbis & & ○ $1 / 2 \mathrm{p}$ & - \\
\hline Scaevola beckii & & ㅇ $1 / 1$ & - \\
\hline Scaevola cylindrica Schltr. \& K. Krause & & - & ô $1 / 2$ \\
\hline Scaevola montana & 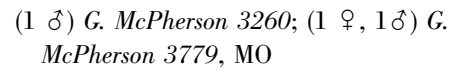 & ๆ 1/1 & ơ $3 / 3$ \\
\hline Scaevola sp. indet. & & ㅇ $1 / 1$ & - \\
\hline Cordyline Comm. ex R. Br. sp. indet. & & - & ○े $2 / 2$ \\
\hline Rhuacophila javanica Blume & J. Munzinger 3046, NOU & ㅇ $1 / 1$ & - \\
\hline $\begin{array}{l}\text { Hugonia penicillanthemum Baill. ex } \\
\text { Pancher \& Sebert }\end{array}$ & G. McPherson 3270, MO & $\uparrow 1 / 1 \mathrm{p}$ & - \\
\hline Tristellateia australasiae A. Rich. & & 아 $1 / 1 \mathrm{p}$ & - \\
\hline Melochia odorata L. f. & G. McPherson 3267, MO & 우 $2 / 5(1 / 2 \mathrm{p})$ & ○ $2 / 3$ \\
\hline $\begin{array}{l}\text { Melastoma malabathricum L. subsp. } \\
\text { malabathricum }\end{array}$ & & ㅇ $4 / 4$ & ô $1 / 1$ \\
\hline Cloezia floribunda Brongn. \& Gris & & - & ot $2 / 2$ \\
\hline Melaleuca quinquenervia & & ㅇ $4 / 11(3 / 10 \mathrm{p})$ & ๙ $3 / 36$ \\
\hline Metrosideros operculata & G. McPherson 3786, MO & o $1 / 3 \mathrm{p}$ & - \\
\hline Sannantha leratii & & 우 $3 / 3(1 / 1 \mathrm{p})$ & ô $13 / 24$ \\
\hline Syzygium P. Browne ex Gaertn. sp. indet. & & ㅇ $1 / 2(1 / 1 \mathrm{p})$ & ô $1 / 1$ \\
\hline Tristaniopsis calobuxus Brongn. \& Gris & & ㅇ $1 / 1 \mathrm{p}$ & ô $1 / 1$ \\
\hline Ludwigia octovalvis subsp. octovalvis & G. McPherson 3785, MO & q $2 / 2$ & ô $1 / 1$ \\
\hline Grevillea R. Br. ex Knight sp. indet. & & - & $\hat{\sigma} 1 / 1$ \\
\hline Stenocarpus phyllodineus & G. McPherson 3317, MO & ㅇ $1 / 2$ & o $1 / 1$ \\
\hline
\end{tabular}


Table 1. Continued.

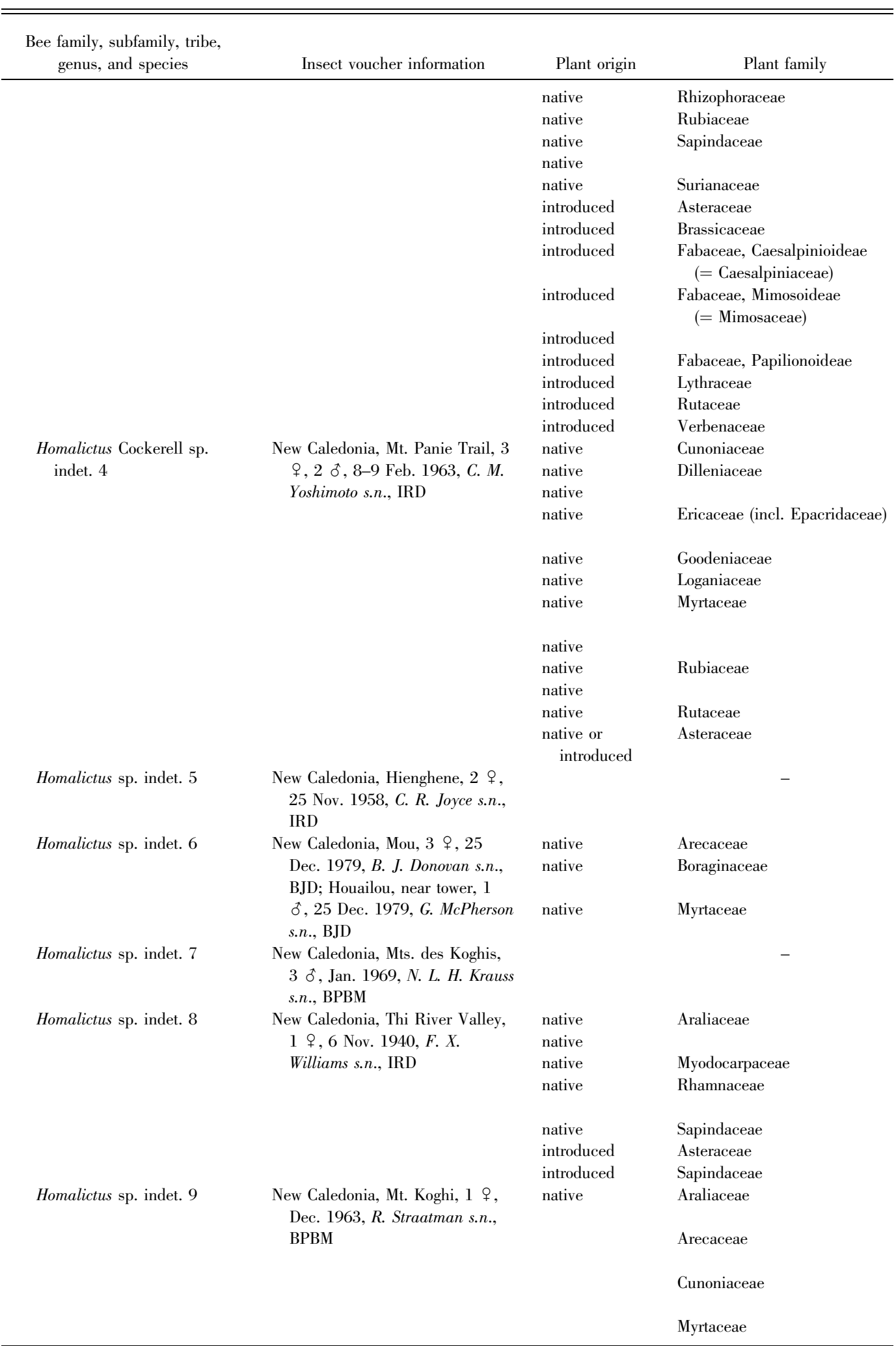


Table 1. Continued, Extended.

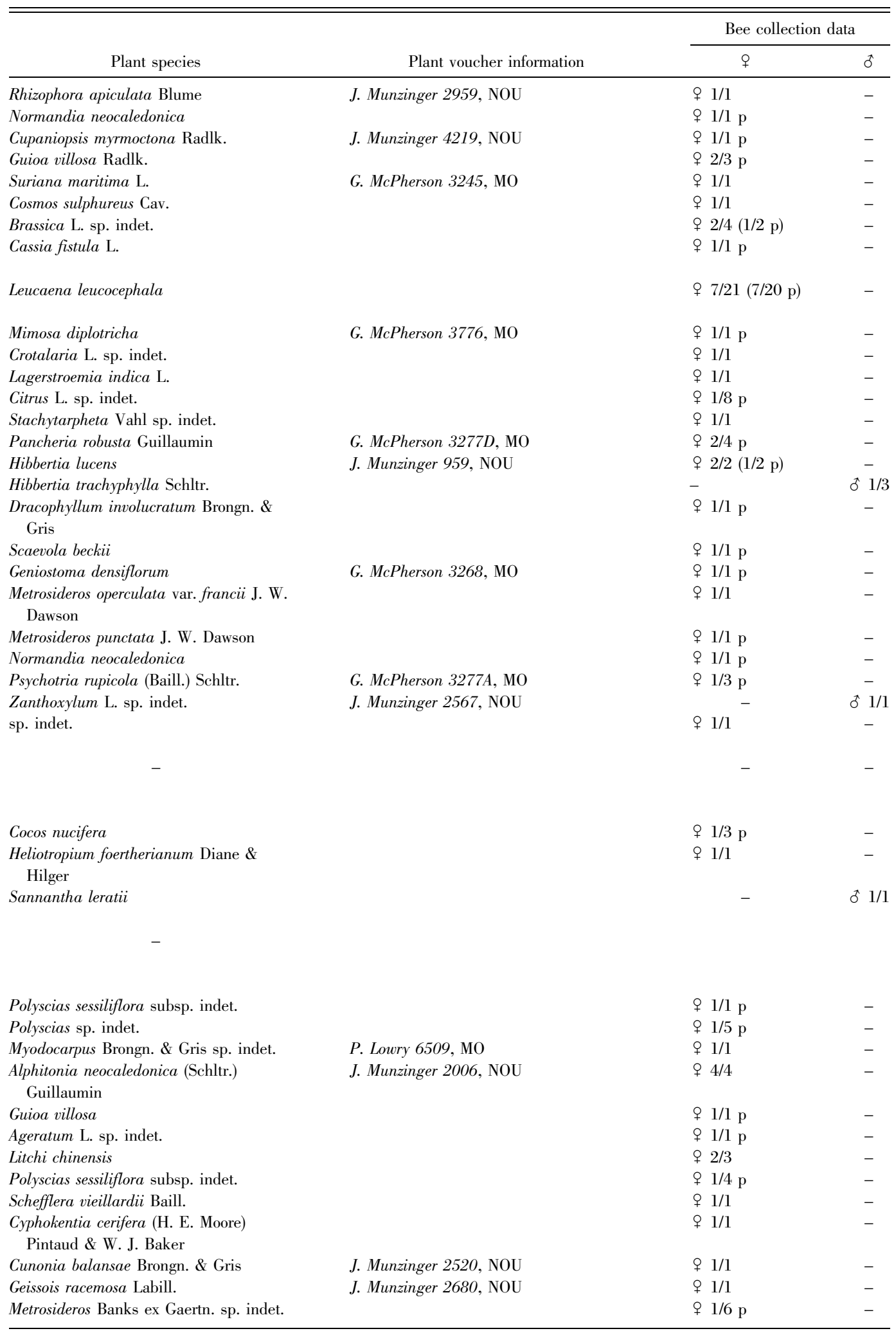


Table 1. Continued.

\begin{tabular}{|c|c|c|c|}
\hline $\begin{array}{l}\text { Bee family, subfamily, tribe, } \\
\text { genus, and species }\end{array}$ & Insect voucher information & Plant origin & Plant family \\
\hline Homalictus sp. indet. 10 & $\begin{array}{l}\text { New Caledonia, Col de Roussettes, } \\
1 \text { }, \text {, }-6 \text { Feb. 1963, G. Kuschel } \\
\text { s.n., BPBM }\end{array}$ & & - \\
\hline Homalictus sp. indet. 11 & $\begin{array}{l}\text { New Caledonia, Île.Mouac, } 1 \text { ô, } \\
19 \text { Oct. 1958, C. R. Joyce s.n., } \\
\text { IRD }\end{array}$ & & - \\
\hline \multirow{10}{*}{$\begin{array}{l}\text { Lasioglossum polygoni } \\
\quad \text { (Cockerell, 1929), subsp. } \\
\text { indet. 1, subg. Chilalictus }\end{array}$} & \multirow{10}{*}{$\begin{array}{l}\text { New Caledonia, Col d'Amieu, } 21 \\
\text { July 1977, A. Delobeli s.n., } \uparrow \\
\text { holotype and } 2 \text { q paratypes, } \\
\text { MNHNP }\end{array}$} & native & Araliaceae \\
\hline & & native & Arecaceae \\
\hline & & native & Cunoniaceae \\
\hline & & native & Dilleniaceae \\
\hline & & native & Ericaceae (incl. Epacridaceae) \\
\hline & & native & Melastomataceae \\
\hline & & native & Myrtaceae \\
\hline & & native & Phellinaceae \\
\hline & & introduced & Sapindaceae \\
\hline & & introduced & Verbenaceae \\
\hline \multirow[t]{14}{*}{$\begin{array}{l}\text { Lasioglossum polygoni subsp. } \\
\text { indet. } 2 \text {, subg. Chilalictus }\end{array}$} & \multirow[t]{14}{*}{$\begin{array}{l}\text { New Caledonia, Haute-Ni, } 1 \text {, } \\
23 \text { Oct. 2004, J. Munzinger s.n., } \\
\text { MNHNP }\end{array}$} & $\begin{array}{l}\text { native } \\
\text { native } \\
\text { native }\end{array}$ & Araliaceae \\
\hline & & native & Cunoniaceae \\
\hline & & native & Dilleniaceae \\
\hline & & native & Elaeocarpaceae \\
\hline & & native & \\
\hline & & native & Ericaceae (incl. Epacridaceae) \\
\hline & & native & Goodeniaceae \\
\hline & & native & Myrtaceae \\
\hline & & native & Sapindaceae \\
\hline & & native & \\
\hline & & native & \\
\hline & & introduced & Asteraceae \\
\hline & & introduced & $\begin{array}{l}\text { Fabaceae, Mimosoideae } \\
\text { (= Mimosaceae })\end{array}$ \\
\hline & & introduced & Sapindaceae \\
\hline \multirow{6}{*}{$\begin{array}{l}\text { Lasioglossum polygoni subsp. } \\
\text { indet. } 3 \text {, subg. Chilalictus }\end{array}$} & \multirow{5}{*}{$\begin{array}{l}\text { New Caledonia, Bourail, } 27 \text { May } \\
\text { 1927, T. D. A. Cockerell s.n., } 1 \\
\text { † holotype, MNHNP }\end{array}$} & native & Asteraceae \\
\hline & & native & Elaeocarpaceae \\
\hline & & native & Violaceae \\
\hline & & introduced & Malvaceae \\
\hline & & & Polygonaceae \\
\hline & & & Solanaceae \\
\hline \multirow{6}{*}{$\begin{array}{l}\text { Lasioglossum Curtis sp. indet. } \\
\quad \text { 4, subg. Chilalictus }\end{array}$} & \multirow{6}{*}{$\begin{array}{l}\text { New Caledonia, Forêt de Sailles, } 1 \\
\text { \&, } 9 \text { Dec. 2001, J. Munzinger } \\
\text { s.n., MNHNP }\end{array}$} & \multirow[t]{6}{*}{ native } & Apocynaceae \\
\hline & & & Araliaceae \\
\hline & & & Dilleniaceae \\
\hline & & & Ericaceae (incl. Epacridaceae) \\
\hline & & & Liliaceae \\
\hline & & & Phellinaceae \\
\hline $\begin{array}{l}\text { Lasioglossum sp. indet. 5, } \\
\text { subg. Chilalictus }\end{array}$ & $\begin{array}{l}\text { New Caledonia, Mt. Khogis, } 22 \\
\text { Dec. 1992, M. E. Irwin \& D. W. } \\
\text { Webb s.n., INHS-96,625 (1 ९), } \\
\text { INHS-96,607 (1 ठ })\end{array}$ & & - \\
\hline
\end{tabular}


Table 1. Continued, Extended.

\begin{tabular}{|c|c|c|c|}
\hline \multirow[b]{2}{*}{ Plant species } & \multirow[b]{2}{*}{ Plant voucher information } & \multicolumn{2}{|c|}{ Bee collection data } \\
\hline & & $q$ & $\hat{0}$ \\
\hline- & & - & - \\
\hline- & & - & - \\
\hline $\begin{array}{l}\text { Polyscias dioica (Vieill. ex Pancher \& } \\
\text { Sebert) Harms }\end{array}$ & J. Munzinger $1732, \mathrm{NOU}$ & 우 $3 / 3$ & - \\
\hline Cyphokentia cerifera & & 우 $2 / 2$ & - \\
\hline Geissois racemosa & J. Munzinger 2680, NOU & o $4 / 4$ & - \\
\hline Hibbertia lucens & (1 \&) J. Munzinger 959, NOU & ㅇ $2 / 2$ & - \\
\hline $\begin{array}{l}\text { Dracophyllum ramosum Pancher ex } \\
\text { Brongn. \& Gris }\end{array}$ & J. Munzinger 2043, NOU & - & ô $1 / 1$ \\
\hline $\begin{array}{l}\text { Melastoma malabathricum subsp. } \\
\text { malabathricum }\end{array}$ & & ๆ 13/13 (1/14 p) & - \\
\hline $\begin{array}{l}\text { Myrtastrum rufopunctatum (Pancher ex } \\
\text { Brongn. \& Gris) Burret }\end{array}$ & & ๆ $1 / 1$ & - \\
\hline Phelline lucida Vieill. ex Baill. & J. Munzinger 2502, NOU & 우 $2 / 2$ & - \\
\hline Litchi chinensis & & ㅇ $1 / 1 \mathrm{p}$ & - \\
\hline Lantana L. sp. indet & & 우 $1 / 3$ & - \\
\hline Polyscias dioica & J. Munzinger $1732, \mathrm{NOU}$ & ๆ $1 / 1$ & - \\
\hline Polyscias sessiliflora subsp. indet. & & 아 $1 / 2 \mathrm{p}$ & - \\
\hline Polyscias sp. indet. & & ㅇ $3 / 12(3 / 11 \mathrm{p})$ & - \\
\hline Pancheria sebertii Guillaumin & & 의 $1 / 1$ & - \\
\hline Hibbertia nana Däniker & & ㅇ $1 / 1$ & - \\
\hline Elaeocarpus dognyensis Guillaumin & & o $1 / 1$ & - \\
\hline Elaeocarpus speciosus Brongn. \& Gris. & J. Munzinger $3058, \mathrm{NOU}$ & ㅇ $2 / 2$ & - \\
\hline Styphelia cf. cymbulae Spreng. & J. Munzinger 2495, NOU & 우 $1 / 1$ & - \\
\hline Scaevola beckii & & ㅇ $2 / 3(1 / 1 \mathrm{p})$ & - \\
\hline Syzygium quadrangulare & & q $1 / 2 \mathrm{p}$ & - \\
\hline Cupaniopsis oedipoda Radlk. & G. McPherson 3227, MO & 아 $1 / 1 \mathrm{p}$ & - \\
\hline Cupaniopsis Radlk. sp. indet. & G. McPherson 2744, MO & o $1 / 2 \mathrm{p}$ & - \\
\hline Guioa villosa & G. McPherson 3238, MO & q $1 / 1 \mathrm{p}$ & - \\
\hline Ageratum conyzoides $\mathrm{L}$. & & ㅇ $4 / 19 \mathrm{p}$ & - \\
\hline Leucaena leucocephala & G. McPherson 3237, MO & @ $1 / 1 \mathrm{p}$ & - \\
\hline Litchi chinensis & & ㅇ $3 / 9(1 / 3 \mathrm{p})$ & - \\
\hline Blumea lacera & & ㅇ $1 / 2(1 / 1 \mathrm{p})$ & - \\
\hline Elaeocarpus angustifolius & & - & ô $1 / 1$ \\
\hline Agatea longipedicellata & J. Munzinger 396, NOU & 우 $1 / 1$ & - \\
\hline Sida acuta & & o $1 / 22(1 / 20 \mathrm{p})$ & - \\
\hline Antigonon leptopus Hook. \& Arn. & & ㅇ $2 / 2$ & - \\
\hline Polygonum L. sp. indet. & & 우 $1 / 1$ & - \\
\hline Solanum torvum & & ㅇ $1 / 4(1 / 3 \mathrm{p})$ & - \\
\hline Parsonsia R. Br. sp. indet. & J. Munzinger 4051, NOU & - & ot $1 / 1$ \\
\hline Polyscias dioica & J. Munzinger $1732, \mathrm{NOU}$ & 우 $2 / 2$ & - \\
\hline Hibbertia nana & J. Munzinger $1649, \mathrm{NOU}$ & ㅇ $2 / 2$ & - \\
\hline Dracophyllum involucratum & F. Tronchet $609, \mathrm{NOU}$ & 우 $3 / 3$ & - \\
\hline Rhuacophila javanica & Th. Le Borgne 39, NOU & 우 $3 / 3$ & - \\
\hline Phelline lucida & J. Munzinger 2502, NOU & 우 $1 / 1$ & - \\
\hline 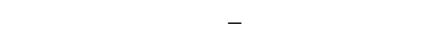 & & - & - \\
\hline
\end{tabular}


Table 1. Continued.

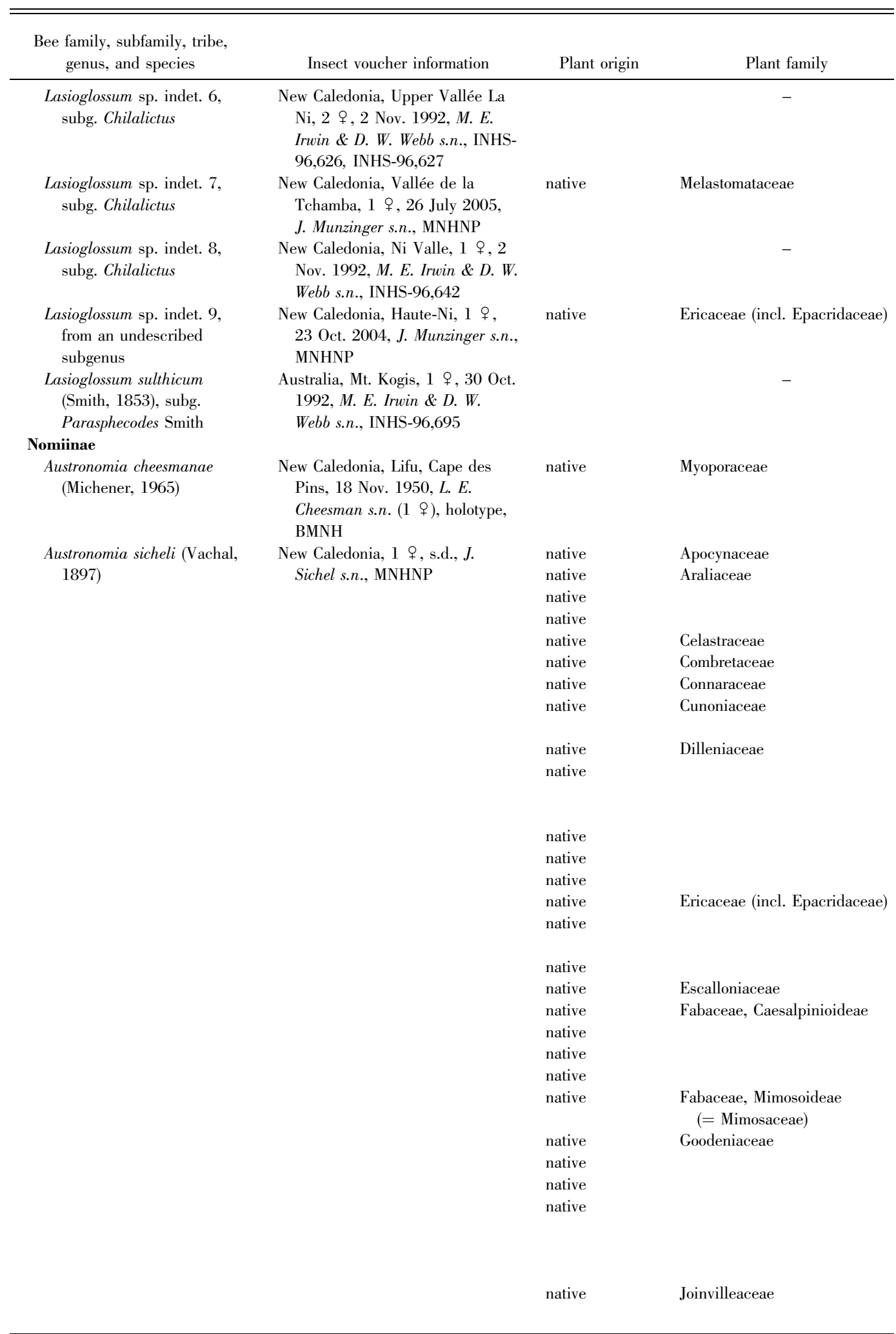


Table 1. Continued, Extended.

\begin{tabular}{|c|c|c|c|}
\hline \multirow[b]{2}{*}{ Plant species } & \multirow[b]{2}{*}{ Plant voucher information } & \multicolumn{2}{|c|}{ Bee collection data } \\
\hline & & q & $\hat{0}$ \\
\hline- & & - & - \\
\hline $\begin{array}{l}\text { Melastoma malabathricum subsp. } \\
\text { malabathricum }\end{array}$ & & ๆ $1 / 1$ & ô $1 / 1$ \\
\hline 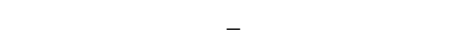 & & - & - \\
\hline Styphelia cf. cymbulae & J. Munzinger 2495, NOU & ๆ $1 / 1$ & - \\
\hline- & & - & - \\
\hline Myoporum crassifolium G. Forst. & & ㅇ $1 / 1$ & - \\
\hline Parsonsia crebriflora Baill. & J. Munzinger 1733, NOU & q 6/6 & - \\
\hline Polyscias sessiliflora subsp. indet. & & ㅇ $1 / 5 \mathrm{p}$ & - \\
\hline Polyscias sp. indet. & & 우 $1 / 5(1 / 3 \mathrm{p})$ & - \\
\hline sp. indet. 1 & & ㅇ $1 / 2$ & - \\
\hline Peripterygia marginata & G. McPherson 3284, MO & ㅇ $1 / 1$ & - \\
\hline $\begin{array}{l}\text { Lumnitzera racemosa Willd. } \\
\text { sp. indet. } 1\end{array}$ & J. Munzinger 2668, NOU & $\stackrel{-}{+} 2 / 3 p$ & $\begin{array}{c}\text { o } 2 / 2 \\
-\end{array}$ \\
\hline $\begin{array}{l}\text { Codia discolor (Brongn. \& Gris) } \\
\text { Guillaumin }\end{array}$ & J. Munzinger 2651, NOU & q $2 / 2 \mathrm{p}$ & - \\
\hline Hibbertia bouletii Veillon & & $? 1 / 1$ & $? 1 / 1$ \\
\hline Hibbertia lucens & $\begin{array}{l}(4 \text { p) G. McPherson } 3272, \mathrm{MO} ;(4 \text { ㅇ }) \mathrm{J} \\
\text { Munzinger } 959 ;(1 \text { 이 }) \mathrm{J} . \text { Munzinger } \\
\text { 2527, NOU }\end{array}$ & ๆ $6 / 9(6 / 6 p)$ & - \\
\hline Hibbertia pancheri & & ㅇ $1 / 1$ & - \\
\hline Hibbertia pulchella & & ㅇ $2 / 2$ & - \\
\hline Hibbertia sp. indet. & (2 ㅇ p) J. C. Bradford 1116, MO & ㅇ $3 / 9(3 / 7 \mathrm{p})$ & - \\
\hline Dracophyllum verticillatum & & ㅇ $0 / 1$ & - \\
\hline $\begin{array}{l}\text { Styphelia pancheri (Brongn. \& Gris) F. } \\
\text { Muell. }\end{array}$ & J. Munzinger 395, NOU & ๆ $1 / 1$ & - \\
\hline Styphelia cf. cymbulae & J. Munzinger 2495, NOU & q $2 / 2$ & - \\
\hline Argophyllum montanum Schltr. & G. McPherson 3269, MO & ㅇ $1 / 1 \mathrm{p}$ & - \\
\hline Cassia fistula & & o $1 / 3 \mathrm{p}$ & - \\
\hline Cassia L. sp. indet. & & 우 $3 / 4(3 / 3 \mathrm{p})$ & - \\
\hline Storckiella pancheri Baill. & & ㅇ $1 / 1 \mathrm{p}$ & - \\
\hline Storckiella Seem. sp. indet. & & 우 $2 / 4(2 / 3 \mathrm{p})$ & - \\
\hline Acacia spirorbis & & $\uparrow 2 / 2 \mathrm{p}$ & ô $1 / 1$ \\
\hline Scaevola beckii & & 우 $2 / 3(1 / 1 \mathrm{p})$ & - \\
\hline Scaevola cylindrica & (19, 1 đ ) G. McPherson 2300, MO & ㅇ $2 / 2$ & o $2 / 3$ \\
\hline Scaevola erosa Guillaumin ex I. H. Müll. & & q $1 / 1$ & - \\
\hline Scaevola montana & 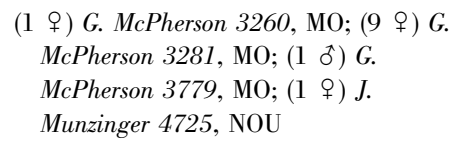 & ㅇ $5 / 12$ & ○ $2 / 2$ \\
\hline $\begin{array}{l}\text { Joinvillea Gaudich. ex Brongn. \& Gris sp. } \\
\text { indet. }\end{array}$ & & ㅇ $1 / 2 \mathrm{p}$ & - \\
\hline
\end{tabular}


Table 1. Continued.

\begin{tabular}{|c|c|c|c|}
\hline $\begin{array}{l}\text { Bee family, subfamily, tribe, } \\
\text { genus, and species }\end{array}$ & Insect voucher information & Plant origin & Plant family \\
\hline & & native & Loganiaceae \\
\hline & & native & Malpighiaceae \\
\hline & & native & Melastomataceae \\
\hline & & native & \\
\hline & & native & Myodocarpaceae \\
\hline & & native & Myrsinaceae \\
\hline & & native & Myrtaceae \\
\hline & & native & \\
\hline & & native & \\
\hline & & native & \\
\hline & & native & \\
\hline & & native & \\
\hline & & native & \\
\hline & & native & \\
\hline & & native & \\
\hline & & native & \\
\hline & & native & \\
\hline & & native & \\
\hline & & native & \\
\hline & & native & \\
\hline & & native & \\
\hline & & native & \\
\hline & & native & Proteaceae \\
\hline & & native & \\
\hline & & native & \\
\hline & & native & \\
\hline & & native & Rhamnaceae \\
\hline & & native & Rubiaceae \\
\hline & & native & Sapindaceae \\
\hline & & native & \\
\hline & & native & Violaceae \\
\hline & & introduced & Anacardiaceae \\
\hline & & introduced & Asteraceae \\
\hline & & introduced & Euphorbiaceae \\
\hline & & introduced & $\begin{array}{l}\text { Fabaceae, Mimosoideae } \\
\text { (= Mimosaceae })\end{array}$ \\
\hline & & introduced & \\
\hline & & introduced & Fabaceae, Papilionoideae \\
\hline & & introduced & Lamiaceae \\
\hline & & introduced & Myrtaceae \\
\hline & & introduced & \\
\hline & & introduced & Phrymaceae \\
\hline & & introduced & Sapindaceae \\
\hline & & introduced & Solanaceae \\
\hline & & introduced & \\
\hline & & introduced & Verbenaceae \\
\hline & & $\begin{array}{l}\text { native or } \\
\text { introduced }\end{array}$ & Convolvulaceae \\
\hline $\begin{array}{l}\text { Austronomia (L.) Link sp. } \\
\quad \text { indet. } 3\end{array}$ & $\begin{array}{l}\text { New Caledonia, Mt. Do, } 1 \text { } 9,15 \\
\text { Dec. } 2000, \text { B. J. Donovan s.n., } \\
\text { BJD }\end{array}$ & introduced & Fabaceae, Papilionoideae \\
\hline Austronomia sp. indet. 4 & $\begin{array}{l}\text { New Caledonia, Loyalty Islands, } \\
\text { Lifu Island, } 1 \text { +, 26-27 Mar. } \\
\text { 1968, J. L. Gressit \& T. C. Maa } \\
\text { s.n., BPBM }\end{array}$ & & - \\
\hline
\end{tabular}


Table 1. Continued, Extended.

\begin{tabular}{|c|c|c|c|}
\hline \multirow[b]{2}{*}{ Plant species } & \multirow[b]{2}{*}{ Plant voucher information } & \multicolumn{2}{|c|}{ Bee collection data } \\
\hline & & q & $\hat{\sigma}$ \\
\hline Geniostoma densiflorum & G. McPherson 3282, MO & q $1 / 3$ & - \\
\hline Tristellateia australasiae & & 우 $1 / 1$ & - \\
\hline Melastoma denticulatum Labill. & & q $2 / 5(1 / 3 \mathrm{p})$ & - \\
\hline $\begin{array}{l}\text { Melastoma malabathricum subsp. } \\
\text { malabathricum }\end{array}$ & & 우 $4 / 5(1 / 2 \mathrm{p})$ & - \\
\hline Myodocarpus fraxinifolius & & ㅇ $2 / 1(2 / 2 \mathrm{p})$ & ô $1 / 3$ \\
\hline Tapeinosperma oblongifolium $\mathrm{Mez}$ & J. Munzinger 1954, NOU & & \\
\hline Cloezia artensis (Montrouz.) P. S. Green & (3 ㅇ, 4 ठ) G. McPherson 3258, MO & 우 $1 / 3$ & ô $3 / 5$ \\
\hline Cloezia Brongn. \& Gris sp. indet. & & ㅇ $1 / 4$ & ô $1 / 1$ \\
\hline Melaleuca gnidioides Brongn. \& Gris & & 우 $1 / 2 \mathrm{p}$ & - \\
\hline Melaleuca quinquenervia & & ㅇ $3 / 4(2 / 3 \mathrm{p})$ & ô $3 / 8$ \\
\hline Myrtastrum rufopunctatum & & 우 $1 / 1$ & - \\
\hline Myrtus L. sp. indet. & & - & of $1 / 73$ \\
\hline Sannantha leratii & & ㅇ $9 / 14$ & ô $3 / 17$ \\
\hline Sannantha Peter G. Wilson sp. indet. & J. Munzinger $456, \mathrm{NOU}$ & - & ô $1 / 1$ \\
\hline Syzygium lateriflorum Brongn. \& Gris & & $3 / 12(1 / 3 \mathrm{p})$ & o $1 / 1$ \\
\hline Syzygium quadrangulare & & ㅇ $1 / 2(1 / 1 \mathrm{p})$ & - \\
\hline Tristaniopsis calobuxus & & 아 $2 / 5$ & ô $3 / 10$ \\
\hline Tristaniopsis glauca Brongn. \& Gris & & ㅇ $1 / 1$ & - \\
\hline Tristaniopsis vieillardii Brongn. \& Gris & J. Munzinger 4679, NOU & ㅇ $1 / 1$ & - \\
\hline $\begin{array}{l}\text { Uromyrtus emarginata (Panch. ex Brongn. } \\
\text { \& Gris) Burret }\end{array}$ & J. Munzinger 394, NOU & $\uparrow 1 / 1$ & - \\
\hline sp. indet. 1 & & † $1 / 1 \mathrm{p}$ & - \\
\hline sp. indet. 2 & J. C. Bradford 1115, MO & ㅇ $1 / 2 \mathrm{p}$ & - \\
\hline Grevillea exul Lindl. & G. McPherson 3271, MO & 우 2/8 (2/4 p) & - \\
\hline Stenocarpus milnei Hook. & G. McPherson 3285, МO & 우 2/14 (1/1 p) & o $2 / 4$ \\
\hline Stenocarpus umbelliferus Druce & & 우 $1 / 1$ & - \\
\hline Stenocarpus R. Br. sp. indet. & & ㅇ $1 / 1 \mathrm{p}$ & ô $1 / 6$ \\
\hline Alphitonia neocaledonica & J. Munzinger 2006, NOU & ๆ $1 / 1$ & - \\
\hline Normandia neocaledonica & G. McPherson 3221, MO & ㅇ $1 / 1 \mathrm{p}$ & - \\
\hline Guioa villosa & & ㅇ $2 / 2 \mathrm{p}$ & - \\
\hline Storthocalyx pancheri (Baill.) Radlk. & J. Munzinger 2053, NOU & ? $1 / 1$ & $? 1 / 1$ \\
\hline Agatea longipedicellata & J. Munzinger $320, \mathrm{NOU}$ & 아 $20 / 20 \mathrm{p}$ & o $11 / 11$ \\
\hline Schinus terebinthifolia & $\left(3\right.$ ㅇ $\left.\left[\begin{array}{lll}1 & + & p\end{array}\right]\right)$ G. McPherson 3241 , MO & ㅇ $5 / 9(3 / 3 \mathrm{p})$ & ô $1 / 8$ \\
\hline Ageratum conyzoides & & 우 $3 / 17(3 / 13 \mathrm{p})$ & - \\
\hline Poinsettia sp. indet. & & 우 $2 / 2$ & o $1 / 1$ \\
\hline Leucaena leucocephala & & ㅇ $5 / 12 \mathrm{p}$ & - \\
\hline Mimosa diplotricha & G. McPherson 3776, MO & $1 / 1$ & - \\
\hline Senna occidentalis (L.) Link & & ㅇ $1 / 1 \mathrm{p}$ & - \\
\hline Mentha L. sp. indet. & & ㅇ $1 / 2$ & - \\
\hline Psidium guajava $\mathrm{L}$. & & ㅇ $1 / 8(1 / 5 \mathrm{p})$ & - \\
\hline Syzygium jambolanum (Lam.) DC. & & ㅇ $1 / 2$ & - \\
\hline Mimulus L. sp. indet. & & ㅇ $1 / 1$ & - \\
\hline Litchi chinensis & & ㅇ $1 / 1$ & - \\
\hline Solanum torvum & (1 + p) G. McPherson $3766 B$, MO & ㅇ $3 / 12 \mathrm{p}$ & - \\
\hline sp. indet. 1 & & $q 2 / 13 \mathrm{p}$ & - \\
\hline Stachytarpheta indica & & ㅇ $1 / 1$ & - \\
\hline Ipomoea L. sp. indet. & & ๆ $1 / 2 \mathrm{p}$ & - \\
\hline Senna occidentalis & & ㅇ $1 / 1$ & - \\
\hline
\end{tabular}


Table 1. Continued.

Bee family, subfamily, tribe,
genus, and species

Austronomia sp. indet. 5

Megachilidae

Megachilinae

Lithurgini

Lithurgus scabrosus (Smith, 1859), subg. Lithurgus Berthold

Megachilini

Megachile laticeps Smith, 1853, subg. Aethomegachile Engel \& Baker

Megachile rambutwan Cheesman, 1936, subg. Callomegachile Michener

Megachile umbripenne Smith, 1853, subg. Callomegachile

Megachile albomarginata Smith, 1879, subg.

Eutricharaea Thomson
Ile Aru, Indonesia, Noumea, 1 ô, July 1900, J. J. Walker s.n., MNHNP

Iles Phillipines, Noumea, 1 , July 1957, J. Rageau s.n., MNHNP

Vanuatu, Noumea, $1 \hat{\jmath}$, Aug. 1900, J. J. Walker s.n., MNHNP

Nepal, Noumea, 2 \&, 15 Oct. 1980, B. J. Donovan s.n., BJD

New Caledonia, Mt. Mou, 1 , 12 Mar. 1914, P. D. Montague 541, MNHN native

native or

introduced

native

native

introduced

native

introduced

introduced

introduced

introduced

native

native

native

native

native

native

native

native

native

native

native

native

native

native

native

introduced

introduced

introduced

introduced

introduced

introduced

introduced

introduced

introduced

introduced

introduced

introduced

introduced
Verbenaceae

Convolvulaceae

Convolvulaceae

Convolvulaceae

Myrtaceae

Proteaceae

Asteraceae

Fabaceae, Papilionoideae

Polygonaceae

Verbenaceae

Apocynaceae

Araliaceae

Connaraceae

Dilleniaceae

Goodeniaceae

Myrtaceae

Proteaceae

Violaceae

Asteraceae

Fabaceae, Mimosoideae (= Mimosaceae)

Lamiaceae

Myrtaceae

Tiliaceae

Verbenaceae 
Table 1. Continued, Extended.

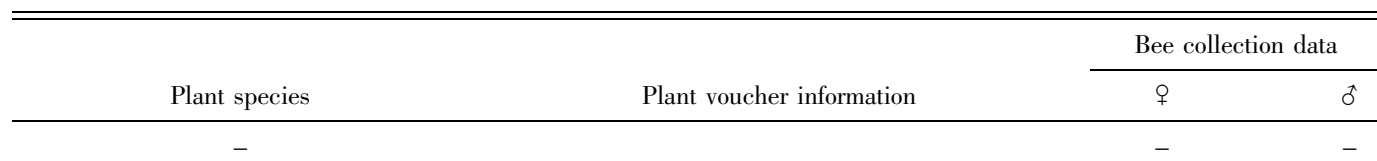

Ipomoea pes-caprae (L.) R. Br.

sp. indet. 1

Hibbertia podocarpifolia

Hibbertia lucens

(2 ㅇ) J. Munzinger 959, NOU; (1 ㅇ) J. Munzinger 2527, NOU

Hibbertia sp. indet.

Scaevola beckii

Scaevola erosa

Scaevola montana

(2 §̋) G. McPherson 3236, MO;

(1 ㅇ 3 §ै) G. McPherson 3779, MO

Syzygium lateriflorum

Stenocarpus sp. indet.

Agatea longipedicellata

Agatea rufotomentosa (Baker f.) J.

Munzinger

Ageratum sp. indet.

Bidens L. sp. indet.

Cosmos Cav. sp. indet.

Tridax procumbens

Albizia Durazz. sp. indet.

J. Munzinger 320, 396, NOU

J. Munzinger 370, 371, NOU

\begin{tabular}{|c|c|}
\hline 우 1/7 (1/4 p) & of $1 / 3$ \\
\hline- & ○े $2 / 8$ \\
\hline † $1 / 2(1 / 1 \mathrm{p})$ & - \\
\hline- & \\
\hline
\end{tabular}

ㅇ $1 / 1 \mathrm{p}$

우 $1 / 1$

우 $1 / 1 \mathrm{p}$

ㅇ $1 / 1 \mathrm{p}$

ㅇ $1 / 1 \mathrm{p}$

ㅇ $1 / 2 \mathrm{p}$

ㅇ $4 / 5(2 / 5 \mathrm{p})$

우 $1 / 1$

아 $1 / 1 \mathrm{p}$

아 $2 / 2(1 / 1 \mathrm{p})$

아 $1 / 1 \mathrm{p}$ ○ $3 / 5$

\begin{tabular}{|c|c|}
\hline- & $01 / 1$ \\
\hline $1 / 1$ & - \\
\hline $8 / 8$ & o $1 / 1$ \\
\hline
\end{tabular}

ㅇ $2 / 2(1 / 2 \mathrm{p})$

ㅇ $2 / 2(1 / 1 \mathrm{p})$

우 $1 / 3$

우 $1 / 6(1 / 5 \mathrm{p})$

우 $2 / 4 \mathrm{p}$

ㅇ $1 / 1 \mathrm{p}$

ㅇ $1 / 1 \mathrm{p}$

ㅇ $1 / 1 \mathrm{p}$

우 $0 / 1$

ㅇ $1 / 1 \mathrm{p}$

$-$

아 $2 / 4(1 / 1 \mathrm{p})$
$-$

$-$

$-$

$-$

Leucaena leucocephala

Ocimum gratissimum L.

G. McPherson $3267 C$, MO

Psidium guajava

Triumfetta rhomboidea Jacq.

Duranta repens

Stachytarpheta indica

(3 우 [1 ㅇ p] 2 ઠै) G. McPherson 3249 , 
Table 1. Continued.

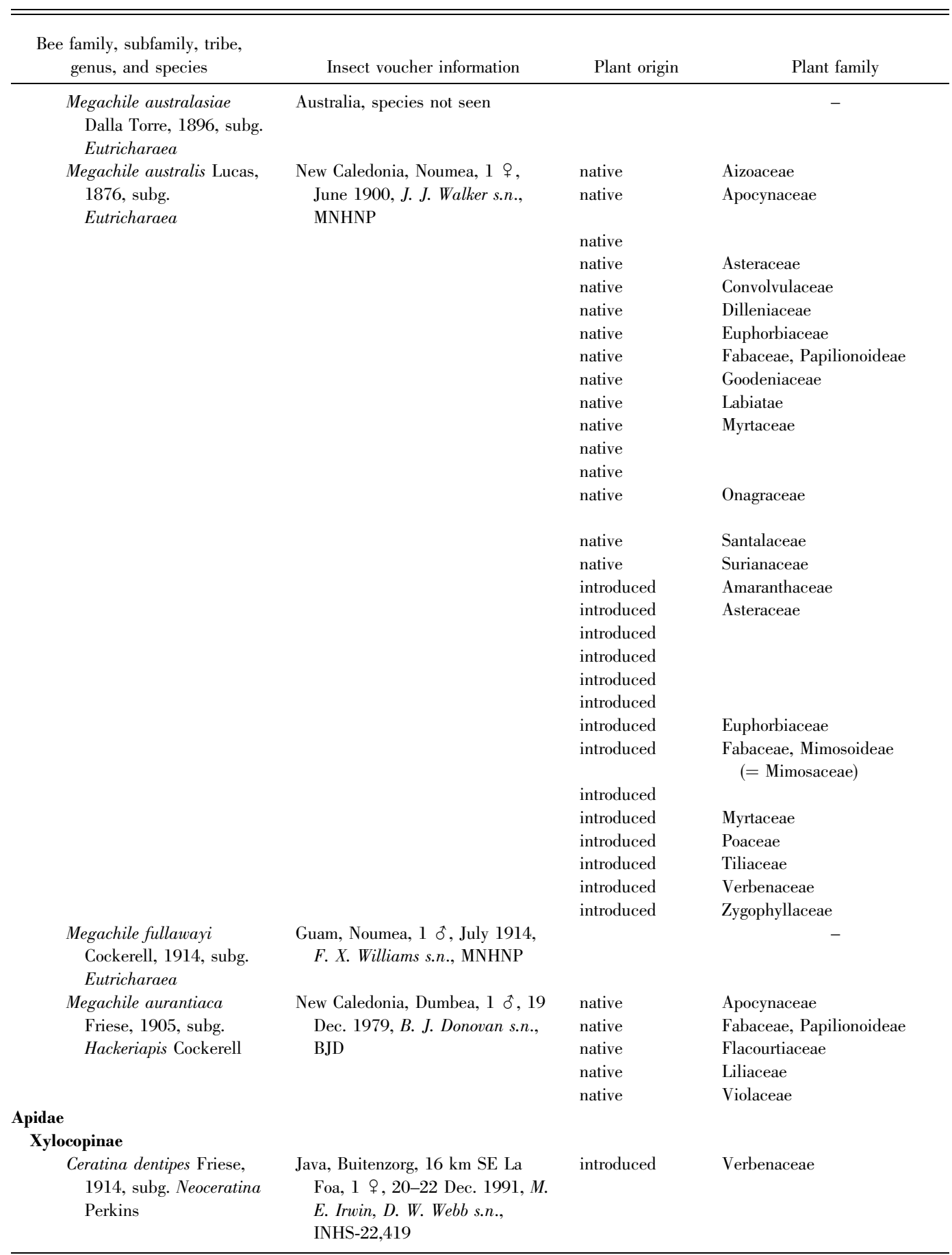


Table 1. Continued, Extended.

\begin{tabular}{|c|c|c|c|}
\hline \multirow[b]{2}{*}{ Plant species } & \multirow[b]{2}{*}{ Plant voucher information } & \multicolumn{2}{|c|}{ Bee collection data } \\
\hline & & 우 & ot \\
\hline - & & - & - \\
\hline
\end{tabular}

Sesuvium portulacastrum L. indet.

Rauvolfia semperflorens

G. McPherson 3235, MO

$\begin{array}{cc}- & \text { के } 1 / 1 \\ \text { ㅇ } 6 / 10(2 / 3 \mathrm{p}) & \text { के } 1 / 1 \\ - & \text { के } 2 / 2\end{array}$

Tridax procumbens

Ipomoea pes-caprae

Tetracera billardieri Martelli

Cleistanthus stipitatus (Baill.) Müll. Arg.

Desmodium incanum DC.

J. Munzinger 861, NOU

우 $1 / 1 \mathrm{p}$

o $1 / 1$

Scaevola sericea Vahl

J. C. Bradford 1074, MO

ㅇ $1 / 1$

우 $1 / 1 \mathrm{p}$

Premna serratifolia L.

Melaleuca quinquenervia

Metrosideros operculata

Sannantha sp. indet. (sensu Babingtonia)

Ludwigia octovalvis subsp. octovalvis

Santalum austrocaledonicum Vieill.

Suriana maritima

Achyranthes aspera

Bidens sp. indet.

Cosmos sulphureus

Cosmos sp. indet.

G. McPherson 3786, MO

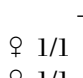

$-$

q $1 / 1$

ㅇ $1 / 1$

G. McPherson $3786, \mathrm{MO}$

ๆ $1 / 1$

(3 ㅇ [1 ㅇ p] 1 §) G. McPherson 3785 ,

우 $1 / 3(1 / 1 \mathrm{p})$

oิ $1 / 1$ MO

J. Munzinger 4518, NOU

G. McPherson 3245, MO

G. McPherson 3246, MO

\begin{tabular}{|c|c|}
\hline - & o $1 / 1$ \\
\hline - & of $2 / 4$ \\
\hline ㅇ $1 / 1$ & ô $1 / 2$ \\
\hline ㅇ $1 / 1$ & - \\
\hline 우 1/1 & of $1 / 1$ \\
\hline ㅇ $1 / 3$ & ô $1 / 1$ \\
\hline - & ธิ $2 / 2$ \\
\hline
\end{tabular}

Sphagneticola trilobata (L.) Pruski

Tridax procumbens

Poinsettia sp. indet.

Leucaena leucocephala

J. Munzinger 405, NOU

(6 우 [3 우 p]) G. McPherson 3247, MO

G. McPherson 3237, MO

G. McPherson 3776, MO

P. Lauri 128, NOU

우 $6 / 10(2 / 3 \mathrm{p}) \quad$ के $1 / 1$

- के $1 / 2$

아 $1 / 1 \mathrm{p} \quad$ के $1 / 1$

ㅇ $1 / 2(1 / 1 \mathrm{p})$

ㅇ $1 / 5 \mathrm{p} \quad-$

ㅇ $2 / 2$

오 $2 / 6(2 / 4 \mathrm{p})$

Triumfetta rhomboidea

Stachytarpheta australis Moldenke

Tribulus cistoides

sp. indet. 1

Nephrodesmus ferrugineus Däniker

Homalium betulifolium Däniker

Dianella sp. indet.

Agatea rufotomentosa

Stachytarpheta australis

Stachytarpheta cayennensis (Rich.) Vahl

\begin{tabular}{|c|c|c|}
\hline \multirow{3}{*}{ J. Munzinger 3011, NOU } & - & ô $1 / 1$ \\
\hline & ㅇ $1 / 1$ & - \\
\hline & ㅇ $1 / 1$ & - \\
\hline & ㅇ $1 / 1$ & - \\
\hline J. Munzinger 371, NOU & 우 $3 / 3$ & o $1 / 1$ \\
\hline
\end{tabular}

ㅇ $1 / 1$

† $2 / 2$

Abbreviations: BJD, Personal collection of Barry J. Donovan; IRD, Institut de Research pour Développement. 
Table 2. Plant families and the number of native and introduced plant genera and species visited by female bees.

\begin{tabular}{|c|c|c|c|c|c|}
\hline Plant families & $\begin{array}{l}\text { Number of } \\
\text { plant genera }\end{array}$ & $\begin{array}{c}\text { Number of } \\
\text { plant species }\end{array}$ & $\begin{array}{c}\text { Number of } \\
\text { bee species } \\
\text { with females } \\
\text { visiting }\end{array}$ & $\begin{array}{l}\text { Number of } \\
\text { bee species } \\
\text { with females } \\
\text { carrying pollen }\end{array}$ & $\begin{array}{c}\text { Number of } \\
\text { plant species from } \\
\text { which pollen } \\
\text { was carried }\end{array}$ \\
\hline \multicolumn{6}{|l|}{ Native plants } \\
\hline Aizoaceae & 1 & 1 & 1 & 0 & 0 \\
\hline Apocynaceae & 3 & 4 & 3 & 2 & 2 \\
\hline Araliaceae & 4 & 7 & 8 & 6 & 4 \\
\hline Arecaceae & 2 & 2 & 5 & $1+1 ?$ & $1+1 ?$ \\
\hline Asteraceae & 2 & 2 & 4 & 2 & 2 \\
\hline Boraginaceae & 1 & 1 & 1 & 0 & 0 \\
\hline Celastraceae & 1 & 1 & 2 & 0 & 0 \\
\hline Connaraceae & 1 & 1 & 2 & 2 & 1 \\
\hline Convolvulaceae & 1 & 1 & 2 & 0 & 0 \\
\hline Cunoniaceae & 4 & 9 & 7 & $4+1 ?$ & $4+1 ?$ \\
\hline Dilleniaceae & 2 & 10 & 9 & $6+1 ?$ & $5+2 ?$ \\
\hline Elaeocarpaceae & 1 & 2 & 1 & 0 & 0 \\
\hline Ericaceae & 2 & 3 & 7 & 1 & 1 \\
\hline Escalloniaceae & 1 & 1 & 1 & 1 & 1 \\
\hline Euphorbiaceae & 1 & 2 & 2 & 0 & 0 \\
\hline Fabaceae & 5 & 7 & $5^{*}$ & $4 *$ & 6 \\
\hline Caesalpinoidea & 2 & 4 & 1 & 1 & 4 \\
\hline Mimosoideae & 1 & 1 & 3 & 3 & 1 \\
\hline Papilionoideae & 2 & 2 & 2 & 11 & 1 \\
\hline Flacourtiaceae & 1 & 1 & 1 & 1 & 0 \\
\hline Goodeniaceae & 1 & 5 & 6 & 5 & 4 \\
\hline Joinvilleaceae & 1 & 1 & 1 & 1 & 1 \\
\hline Labiatae & 1 & 1 & 1 & 0 & 0 \\
\hline Liliaceae & 2 & 2 & 3 & 0 & 0 \\
\hline Linaceae & 1 & 1 & 1 & 1 & 1 \\
\hline Loganiaceae & 1 & 1 & 3 & 1 & 1 \\
\hline Malpighiaceae & 2 & 2 & 3 & 2 & 2 \\
\hline Malvaceae & 1 & 1 & 1 & 1 & 1 \\
\hline Melastomataceae & 1 & 2 & 4 & 2 & 2 \\
\hline Myodocarpaceae & 1 & 2 & 1 & 1 & 1 \\
\hline Myoporaceae & 1 & 1 & 1 & 0 & 0 \\
\hline Myrsinaceae & 1 & 1 & 1 & 0 & 0 \\
\hline Myrtaceae & 8 & 21 & 9 & $6+1 ?$ & 11 \\
\hline Onagraceae & 1 & 1 & 3 & 2 & 1 \\
\hline Orchidaceae & 1 & 1 & 1 & 0 & 0 \\
\hline Phellinaceae & 1 & 1 & 2 & 0 & 0 \\
\hline Proteaceae & 2 & 5 & 5 & 3 & 4 \\
\hline Rhamnaceae & 1 & 1 & 2 & 0 & 0 \\
\hline Rhizophoraceae & 1 & 1 & 1 & 0 & 0 \\
\hline Rubiaceae & 2 & 2 & 4 & 3 & 2 \\
\hline Sapindaceae & 2 & 4 & 4 & 4 & 4 \\
\hline Surianaceae & 1 & 1 & 1 & 0 & 0 \\
\hline Tiliaceae & 1 & 1 & 1 & 0 & 0 \\
\hline Violaceae & 1 & 2 & 5 & 3 & 2 \\
\hline \multicolumn{6}{|l|}{ Introduced plants } \\
\hline Amaranthaceae & 2 & 2 & 2 & 1 & 1 \\
\hline Anacardiaceae & 1 & 1 & 2 & 2 & 1 \\
\hline Asteraceae & 8 & 12 & 7 & 6 & 6 \\
\hline Brassicaceae & 2 & 2 & 2 & 1 & 1 \\
\hline Euphorbiaceae & 2 & 3 & 3 & 1 & 2 \\
\hline Fabaceae & 8 & 8 & $8^{*}$ & $7 *$ & 6 \\
\hline Caesalpinioideae & 2 & 2 & 2 & 1 & 1 \\
\hline Mimosoideae & 3 & 3 & 6 & 6 & 3 \\
\hline Papilionoideae & 3 & 3 & 4 & 2 & 2 \\
\hline
\end{tabular}


Table 2. Continued.

\begin{tabular}{|c|c|c|c|c|c|}
\hline Plant families & $\begin{array}{l}\text { Number of } \\
\text { plant genera }\end{array}$ & $\begin{array}{c}\text { Number of } \\
\text { plant species }\end{array}$ & $\begin{array}{c}\text { Number of } \\
\text { bee species } \\
\text { with females } \\
\text { visiting }\end{array}$ & $\begin{array}{l}\text { Number of } \\
\text { bee species } \\
\text { with females } \\
\text { carrying pollen }\end{array}$ & $\begin{array}{c}\text { Number of } \\
\text { plant species from } \\
\text { which pollen } \\
\text { was carried }\end{array}$ \\
\hline Lamiaceae & 2 & 2 & 2 & 1 & 1 \\
\hline Lythraceae & 1 & 1 & 1 & 1 & 1 \\
\hline Malvaceae & 1 & 2 & 2 & 2 & 2 \\
\hline Myrtaceae & 3 & 3 & 3 & 3 & 1 \\
\hline Phrymaceae & 1 & 1 & 1 & 0 & 0 \\
\hline Poaceae & 1 & 1 & 1 & 0 & 0 \\
\hline Polygonaceae & 2 & 2 & 1 & 0 & 0 \\
\hline Rutaceae & 1 & 1 & 1 & 1 & 1 \\
\hline Sapindaceae & 1 & 1 & 4 & $2+1 ?$ & $1+1 ?$ \\
\hline Solanaceae & 1 & 3 & 3 & 3 & 3 \\
\hline Tiliaceae & 1 & 1 & 2 & 2 & 1 \\
\hline Verbenaceae & 4 & 7 & 7 & 2 & 2 \\
\hline Zygophyllaceae & 1 & 1 & 1 & 1 & 1 \\
\hline \multicolumn{6}{|c|}{ Native or introduced plants (status uncertain) } \\
\hline Asteraceae & 1 & 1 & 1 & 0 & 0 \\
\hline Convolvulaceae & 1 & 1 & 2 & 2 & 1 \\
\hline
\end{tabular}

? Refers to bee species that carry pollen internally. For example, $4+1$ ? means that females of four species were carrying pollen and females of one species were possibly carrying pollen internally.

* Indicates that in these columns, the numbers for the subfamilies of Fabaceae do not match the totals for Fabaceae as a whole. This is because some species of bees visit more than one subfamily.

the 28 recorded by Donovan (1983) and the 21 of Pauly and Munzinger (2003), there is uncertainty as to the number of bee species that might be truly native and the number that might have colonized the country in historic times through agencies of human transport. But if all 43 species are considered, with 3051 species of flowering plants thought to be native to New Caledonia (Morat et al., 2012), the ratio of species of bees to flowering plants is about 1:71. This is extremely low compared to Australia, which lies $1200 \mathrm{~km}$ to the west, where the ratio is about 1:7 (Donovan, 1983). However, for New Zealand, which is about $1600 \mathrm{~km}$ to the southeast, a recent assessment of 28 native species of bees (Donovan, 2007), and 1612 native species of flowering plants (Allen, 1961; Moore \& Edgar, 1976) gives a ratio of 1:57, which suggests that the comparative relationships of native bees and plants of the two island countries may be rather similar.

Apart from Lithurgus scabrosus Smith, which is found only on Ipomoea pes-caprae (L.) R. Br. (Pauly \& Munzinger, 2003), there appear to be no obvious specializations of any species of bees to foraging on particular flowers, and indeed the ablity of the most common species of bees to forage on at least some introduced flowers suggests that their foraging habits are quite plastic. For the uncommon species of bees, the data are too few to allow judgments.
The 116 native species of flowering plants visited by the females of New Caledonian native bees are just $3.8 \%$ of the 3051 native species of flowering plants. However, because of height from the ground, the density of foliage, and ruggedness of the landscape, many flowers are difficult to reach with insect collecting equipment, and if access were better there is no doubt that bees would be shown to visit many more flowers. Another major point is that for 20 species of bees there are no records of females associated with flowers. A primary reason for the lack of records is that for several species of bees only one or a few males were collected. Also, if females were collected and especially if very few were collected, there may be no flower records. Only half a dozen species of bees can be considered to be common and perhaps occasionally numerous, of which the primary species are listed in Table 3. A survey of flower visitors at 31 sites over Grande Terre by Kato and Kawakita (2004) found that for 541 individual Apoidea, $89.8 \%$ were the introduced honey bee Apis mellifera, followed by the Halictidae (6\%) and Megachilidae (3\%). Colletid bees were not seen. Moreover, honey bees were found in all vegetation types, at every altitude and locality. Kato and Kawakita (2004) concluded that native bees must have been abundant and played an important role in pollination before the immigration of honey bees. They suggest that native bees are now endangered 
Table 3. Species of bees with females visiting native and introduced plant families, genera, and species, ranked by the highest number of plant species visited, or for those with equal scores, thereafter as listed in Table 1. Question marks refer to pollen possibly carried internally; P refers to the kleptoparasitic bees of Lasioglossum, an undescribed subgenus in which pollen is not collected by females.

\begin{tabular}{|c|c|c|c|c|}
\hline \multirow[b]{2}{*}{ Species of bee } & \multicolumn{3}{|c|}{ Numbers of visited plants } & \multirow{2}{*}{$\begin{array}{l}\text { Number of plant species } \\
\text { from which pollen was carried }\end{array}$} \\
\hline & Families & Genera & Species & \\
\hline \multicolumn{5}{|l|}{ Visitors to native plants } \\
\hline Austronomia sicheli & 22 & 32 & 53 & 29 \\
\hline Homalictus risbeci & 18 & 24 & 30 & 15 \\
\hline Homalictus aponi & 18 & 19 & 27 & 15 \\
\hline Lasioglossum polygoni & 12 & 16 & 21 & 9 \\
\hline Megachile albomarginata & 7 & 8 & 14 & 10 \\
\hline Megachile australis & 9 & 10 & 10 & 4 \\
\hline Homalictus sp. indet. 4 & 7 & 8 & 9 & 8 \\
\hline Homalictus sp. indet. 9 & 4 & 6 & 6 & 2 \\
\hline Euhesma sp. indet. 1 & 3 & 3 & 5 & $5 ?$ \\
\hline Homalictus sp. indet. 8 & 4 & 4 & 5 & 3 \\
\hline Lasioglossum sp. indet. 4 & 5 & 5 & 5 & 0 \\
\hline Megachile aurantiaca & 4 & 4 & 4 & 0 \\
\hline Homalictus cocos & 2 & 2 & 2 & 0 \\
\hline Homalictus sp. indet. 6 & 2 & 2 & 2 & 1 \\
\hline Megachile laticeps & 2 & 2 & 2 & 0 \\
\hline Leioproctus pacificus & 1 & 1 & 1 & 0 \\
\hline Palaeorhiza flavomellea & 1 & 1 & 1 & $1 ?$ \\
\hline Lasioglossum sp. indet. 7 & 1 & 1 & 1 & 0 \\
\hline Lasioglossum sp. indet. 9 & 1 & 1 & 1 & $\mathrm{P}$ \\
\hline Lithurgus scabrosus & 1 & 1 & 1 & 0 \\
\hline Megachile umbripenne & 1 & 1 & 1 & 1 \\
\hline \multicolumn{5}{|l|}{ Visitors to introduced plants } \\
\hline Homalictus aponi & 9 & 19 & 24 & 14 \\
\hline Austronomia sicheli & 10 & 13 & 14 & 8 \\
\hline Megachile australis & 7 & 10 & 11 & 5 \\
\hline Megachile albomarginata & 6 & 10 & 10 & 8 \\
\hline Homalictus risbeci & 6 & 9 & 9 & 6 \\
\hline Lasioglossum polygoni & 6 & 7 & 7 & 5 \\
\hline Homalictus cocos & 3 & 3 & 3 & 1 \\
\hline Homalictus sp. indet. 8 & 2 & 2 & 2 & 1 \\
\hline Ceratina dentipes & 1 & 1 & 2 & 0 \\
\hline Euryglossina sp. indet. 1 & 1 & 1 & 1 & $1 ?$ \\
\hline Austronomia sp. indet. 3 & 1 & 1 & 1 & 0 \\
\hline Megachile umbrippenne & 1 & 1 & 1 & 1 \\
\hline \multicolumn{5}{|c|}{ Visitors to native or introduced plants (status uncertain) } \\
\hline Homalictus sp. indet. 4 & 4 & 1 & 1 & 0 \\
\hline Austronomia sicheli & 1 & 1 & 1 & 1 \\
\hline Lithurgus scabrosus & 1 & 1 & 1 & 1 \\
\hline
\end{tabular}

because of competitive pressure from honey bees, and that original plant-pollinator interactions have been altered.

Although direct evidence of competitive interactions between native bees and honey bees has not yet been documented, our findings that most species of native bees are very uncommon strongly support the opinions of Kato and Kawakita (2004). Because honey bees forage for nectar and pollen whenever weather allows them to, and they are extremely polylectic, native bees in tropical New Caledonia are faced with continuous competitive foraging pressure from this introduced bee. Unless honey bee numbers are reduced, perhaps by the future occurrence of the mite Varroa destructor Anderson \& Trueman, the survival of most of the native species of bees in New Caledonia may be uncertain, which would forever obscure the original relationships between them and the native flora. 


\section{Literature Cited}

Allen, H. H. 1961. Flora of New Zealand, Vol. 1. R. E. Owen, Government Printer, Wellington.

Donovan, B. J. 1983. Comparative biogeography of native Apoidea of New Zealand and New Caledonia. GeoJournal 7: $511-516$.

Donovan, B. J. 2007. Apoidea (Insecta: Hymenoptera). In T. K. Crosby (editor), Fauna of New Zealand 57. Manaaki Whenua Press, Landcare Research Ltd., Lincoln.

Groom, S. V. C. \& M. P. Schwarz. 2011. Bees in the Southwest Pacific: Origins, diversity and conservation. Apidologie 42: 759-770.

Irwin, G. 2010. Navigation and seafaring. Pp. 47-72 in I. Lilley (coordinator), Early Human Expansion and Innovation in the Pacific. ICOMOS Theoretical Study, Paris.

Jaffré, T., P. Morat, F. Rigault, J. M. Veillon \& G. Dagostini. 2001. Composition et caractéristiques de la flore indigène de la Nouvelle-Calédonie. IRD, Nouméa, New Caledonia.

Jaffré, T., P. Morat, F. Rigault, J. M. Veillon \& G. Dagostini. 2004. Composition et Caractéristiques de la Flore Indigène de la Nouvelle-Calédonie, 2nd ed. IRD, Nouméa, New Caledonia.

Kato, M. \& A. Kawakita. 2004. Plant-pollinator interactions in New Caledonia influenced by introduced honey bees. Amer. J. Bot. 91: 1814-1827.
Lamaignere, H. 2001. L'apiculture en Nouvelle-Caledonie. Theses pour obtenir le grade de Docteur Veterinaire Diplome D'Etat, Ecole Nationale Veterinaire, Toulouse.

Lowry II, P. P., J. Munzinger, P. Bouchet, H. Géraux, A. Bauer, O. Langrand \& R. A. Mittermeier. 2004. New Caledonia. Pp. 193-197 in R. A. Mittermeier, P. Robles Gil, M. Hoffmann, J. Pilgrim, T. Brooks, C. G. Mittermeier, J. L. Lamoreux \& G. A. B. da Fonseca (editors), Hotspots Revisited: Earth's Biologically Richest and Most Threatened Terrestrial Ecoregions. CEMEX, Mexico City.

Michener, C. D. 2007. Bees of the World, 2nd ed. The Johns Hopkins University Press, Baltimore and London. Moore, L. B. \& E. Edgar. 1976. Flora of New Zealand, Vol. 2. A. R. Shearer, Government Printer, Wellington.

Morat, P., T. Jaffré, F. Tronchet, J. Munzinger, Y. Pillon, J.M. Veillon \& M. Chalopin. 2012. The taxonomic database FLORICAL and characteristics of the indigenous flora of New Caledonia. Adansonia sér. 3, 34: $177-$ 219.

Nielsen, I., J.-N. Labat \& J. Munzinger. 2005. Synopsis of Storckiella Seem. (Leguminosae-Caesalpinioideae) with description of a new species and a new subspecies from New Caledonia. Adansonia sér. 3, 27: 217-230.

Pauly, A. \& J. Munzinger. 2003. Contribution à la connaissance des Hymenoptera Apoidea de NouvelleCalédonie et de leurs relations avec la flore butinée. Ann. Soc. Entomol. Fr. 39: 153-166. 